This item was submitted to Loughborough's Research Repository by the author.

Items in Figshare are protected by copyright, with all rights reserved, unless otherwise indicated.

\title{
Non-Loudon-Fleury Raman scattering in spin-orbit coupled Mott insulators
}

\section{PLEASE CITE THE PUBLISHED VERSION}

https://doi.org/10.1103/physrevb.104.144412

\section{PUBLISHER}

American Physical Society (APS)

VERSION

VoR (Version of Record)

\section{PUBLISHER STATEMENT}

This paper was accepted for publication in the journal Physical Review B and the definitive published version is available at https://doi.org/10.1103/physrevb.104.144412

\section{LICENCE}

CC BY-NC-ND 4.0

\section{REPOSITORY RECORD}

Yang, Yang, Mengqun Li, loannis Rousochatzakis, and Natalia B Perkins. 2021. "Non-loudon-fleury Raman Scattering in Spin-orbit Coupled Mott Insulators". Loughborough University. https://hdl.handle.net/2134/16823419.v1. 


\title{
Non-Loudon-Fleury Raman scattering in spin-orbit coupled Mott insulators
}

\author{
Yang Yang $\odot,{ }^{1}$ Mengqun Li ${ }^{1}$ Ioannis Rousochatzakis $\odot,{ }^{2}$ and Natalia B. Perkins $\oplus^{1}$ \\ ${ }^{1}$ School of Physics and Astronomy, University of Minnesota, Minneapolis, Minnesota 55455, USA \\ ${ }^{2}$ Department of Physics, Loughborough University, Loughborough LE11 3TU, United Kingdom
}

(Received 4 June 2021; accepted 21 September 2021; published 14 October 2021)

\begin{abstract}
We revisit the theory of magnetic Raman scattering in Mott insulators with strong spin-orbit coupling, with a major focus on Kitaev materials. We show that Kitaev materials with bond-anisotropic interactions are generally expected to show both one- and two-magnon responses. It is further shown that, in order to obtain the correct leading contributions to the Raman vertex operator $\mathcal{R}$, one must take into account the precise, photon-assisted microscopic hopping processes of the electrons and that, in systems with multiple hopping paths, $\mathcal{R}$ contains terms beyond those appearing in the traditional Loudon-Fleury theory. Most saliently, a numerical implementation of the revised formalism to the case of the three-dimensional hyperhoneycomb Kitaev material $\beta$ - $\mathrm{Li}_{2} \mathrm{IrO}_{3}$ reveals that the non-Loudon-Fleury scattering terms actually dominate the Raman intensity. In addition, they induce a qualitative modification of the polarization dependence, including, e.g., the emergence of a sharp one-magnon peak at low energies, which is not expected in the traditional Loudon-Fleury theory. This peak is shown to arise from microscopic photon-assisted tunneling processes that are of similar type with the ones leading to the symmetric off-diagonal interaction $\Gamma$ (known to be present in many Kitaev materials), but take the form of a bond-directional magnetic dipole term in the Raman vertex. These results are expected to apply across all Kitaev materials and mark a drastic change of paradigm for the understanding of Raman scattering in materials with strong spin-orbit coupling and multiple exchange paths.
\end{abstract}

DOI: 10.1103/PhysRevB.104.144412

\section{INTRODUCTION}

Raman scattering has proven to be a powerful experimental technique to understand and characterize the physics of strongly correlated systems [1]. Being a sensitive probe to single-particle and multiparticle excitations over sufficiently wide ranges of temperatures and energies, Raman scattering has played an important role in elucidating ground state properties, symmetry, and statistics of magnetic excitations, as well as the strength and nature of the exchange couplings in magnetic insulators with both magnetically ordered and spin liquid ground states [2-25]. In recent years, there has been a series of Raman studies (both experimental and theoretical) on a range of spin-orbit coupled (SOC) Mott insulators, with a view to elucidate the nature of their magnetic excitations (and lattice dynamics) and their proximity to the so-called Kitaev quantum spin liquid ground states [13-34]. Most saliently, the reported Raman scattering data in the Kitaev candidate materials $\alpha-\mathrm{RuCl}_{3}[13,14,17-19]$ and the three-dimensional (3D) iridates $\beta-\mathrm{Li}_{2} \mathrm{IrO}_{3}$ and $\gamma-\mathrm{Li}_{2} \mathrm{IrO}_{3}$ [16], revealed signatures of both multiparticle continua, characteristics of the proximate spin liquid phase, and sharp peaks, characteristic of magnon excitations of the low-temperature ordered phases. These results call for a close reexamination of the Raman scattering theory applied to strong spin-orbit coupled Mott insulators.

The history of understanding of the magnetic Raman scattering goes back to the seminal paper by Fleury and Loudon [35], in which they have identified three main mechanisms for the coupling between light and magnetic excitations: (i) direct coupling of photon to magnon through magnetic-dipole interaction, (ii) indirect electric-dipole coupling, which mixes the spin and orbital motion of the electrons, and (iii) secondorder electric-dipole coupling, which is very similar to an exchange mechanism. The first mechanism (i) is very weak and is usually neglected. The second (ii) is the Elliott-Loudon mechanism [36], in which the Raman scattering from the magnetic degrees of freedom on a single ion proceeds via a pair of allowed electric-dipole transitions through a spin-orbit active intermediate state. In this process, the incident light excites an electron from the ground state to an excited state keeping the $z$ component of the spin unchanged. The spin states with different $z$ components are then mixed in the excited state via the spin-orbit coupling, and a transition back to the ground state but with opposite spin polarization can occur by emitting a Raman photon and a magnon with $\Delta S^{z}= \pm 1$. Traditionally, the Elliott-Loudon process is considered to be the main source of the one-magnon scattering response, and this is indeed the case in systems with weak SOC. The process (ii) also gives rise to two-magnon scattering, but its intensity is several orders of magnitude weaker compared to the one-magnon process.

This brings us to the third mechanism (iii), which is the exchange-scattering mechanism described by the wellknown Loudon-Fleury theory of magnetic Raman scattering in Mott insulators [35]. The basic idea of this theory is that the processes leading to the Raman response from Mott insulators are similar to those leading to the exchange interactions, except that the virtual electron hopping is (partly) 
assisted by photons. Consequently, the Loudon-Fleury Raman operator is proportional to the sum over the individual spin-exchange interactions, weighted by bond-specific, polarization-dependent factors that determine the ability of photons to control the magnitude of the associated electron hopping $[1-3,8,10,11,20,24,35]$. Traditionally, it is considered that the processes involved in this mechanism contribute predominantly to the two-magnon scattering with $\Delta S^{z}=0$, in which a pair of magnons is created or destroyed. This perception follows in part from a concluding remark in the original paper of Loudon and Fleury [35] that "the exchange mechanism discussed here (being proportional to $S_{i}^{+} S_{j}^{-}$) produces magnons in pairs and hence there is no exchange-scattering mechanism for one-magnon scattering." Now we understand that this statement is certainly far from being general and, in particular, it does not apply to the SOC Mott insulators with bond-dependent anisotropic interactions, which naturally give rise to one-magnon response [17,18,30-33,37].

Here we show that in the SOC Mott insulators, the exchange-scattering mechanism (iii) leads to essential contributions beyond the Loudon-Fleury theory, and these non-Loudon-Fleury terms can give rise to a significant onemagnon Raman response, on top of the two-magnon response. Quite remarkably, our numerical calculations for the representative case of $\beta-\mathrm{Li}_{2} \mathrm{IrO}_{3}$ shows that the Raman intensity (both in the one- and the two-magnon channels) is actually dominated by the contribution from the non-Loudon-Fleury terms by at least two orders of magnitude. In addition, these terms give rise to a qualitative modification of the scattering intensity including its polarization dependence. These include a distinctive, one-magnon low-energy peak in the ac polarization channel [37], which is not expected in the traditional Loudon-Fleury theory. As we discuss below, similar results are expected across all Kitaev materials, given that they all share the same local geometry of virtual exchange paths and the same order of magnitude of microscopic hopping and interaction parameters. In this sense, the theoretical framework presented below calls for a general reevaluation of Raman scattering in Kitaev materials of current interest and systems with strong spin-orbit coupling and multiple exchange paths more generally.

The remainder of the paper is organized as follows. In Sec. II, we begin with a brief discussion of the relevant materials: strong SOC Mott insulators in which the magnetic moment $j_{\text {eff }}=1 / 2$ comes from the five electrons (or one hole) on the $t_{2 g}$ orbitals and their effective low-energy description. In Sec. III, we present the main steps of the $\mathcal{T}$-matrix formalism that lead to the microscopic derivation of the Raman operator $\mathcal{R}$. In Sec. IV, we apply this framework to Kitaev materials, and establish the leading contributions to the Raman vertex $\mathcal{R}$ from the same microscopic processes that give rise to the minimal $J-K-\Gamma$ model. These include processes arising from direct hopping, ligand-mediated hopping as well as processes involving both direct and ligand-mediated hopping. We then establish that the latter two types of processes are the ones giving rise to the strong non-Loudon-Fleury contributions to the Raman operator. In Sec. V, we proceed with the application to magnetically ordered states. To that end, we express $\mathcal{R}$ in terms of magnon operators and obtain the expressions for the one- and two-magnon Raman intensities. The numerical implementation of this theory to the three-dimensional Kitaev magnet $\beta$ - $\mathrm{Li}_{2} \mathrm{IrO}_{3}$ is then presented in Sec. VI, where it is demonstrated that the non-Loudon-Fleury terms dominate the Raman intensity by at least two orders of magnitude. Section VII provides a brief summary along with a general perspective of the results. Some of the technical details and auxiliary information are relegated to Appendix.

\section{RELEVANT MATERIALS AND GENERIC LOW-ENERGY DESCRIPTION}

The theory developed below applies to SOC Mott insulators, such as the Kitaev materials with $\mathrm{Ir}^{4+}$ and $\mathrm{Ru}^{3+}$ ions, in which the magnetic moment $j_{\text {eff }}=1 / 2$ comes from the five electrons (or one hole) on the $t_{2 g}$ orbitals due to the strong SOC $[26,28,38-40]$. These include, e.g., the layered compounds $\mathrm{Na}_{2} \mathrm{IrO}_{3}$ [41,42], $\alpha-\mathrm{Li}_{2} \mathrm{IrO}_{3}$ [43], and $\alpha-\mathrm{RuCl}_{3}$ [44-47], as well as the three-dimensional (3D) iridates $\beta$ - $\mathrm{Li}_{2} \mathrm{IrO}_{3}$ [48-50] and $\gamma-\mathrm{Li}_{2} \mathrm{IrO}_{3}$ [51,52], for which most of the experimental Raman data has been reported so far.

The minimal electronic Hamiltonian of such SOC Mott insulators contains the following terms:

$$
\mathcal{H}=\mathcal{H}_{\text {int }}+\mathcal{H}_{\mathrm{pd}}+\mathcal{H}_{\mathrm{sOC}}+\mathcal{H}_{t},
$$

where $\mathcal{H}_{\text {int }}$ is the interaction part of the three-orbital Hubbard Hamiltonian, $\mathcal{H}_{\mathrm{pd}}=\Delta_{p d} \sum_{i, \sigma} n_{i \sigma}$ is the charge-transfer Hamiltonian (where $\Delta_{p d}$ stands for the charge-transfer energy of one electron from the magnetic ion to the ligand ion), and $\mathcal{H}_{\mathrm{SOC}}$ described the on-site SOC, and $\mathcal{H}_{t}$ stands for the hopping. Specifically,

$$
\begin{aligned}
\mathcal{H}_{\text {int }}= & \sum_{i}\left(U_{1} \sum_{\alpha} n_{i \alpha \uparrow} n_{i \alpha \downarrow}+\frac{1}{2}\left(U_{2}-J_{H}\right) \sum_{\alpha \neq \alpha^{\prime}, \sigma} n_{i \alpha \sigma} n_{i \alpha^{\prime} \sigma}\right. \\
& +U_{2} \sum_{\alpha \neq \alpha^{\prime}} n_{i \alpha \uparrow} n_{i \alpha^{\prime} \downarrow}+J_{H} \sum_{\alpha \neq \alpha^{\prime}} d_{i \alpha \uparrow}^{\dagger} d_{i \alpha \downarrow}^{\dagger} d_{i \alpha^{\prime} \downarrow} d_{i \alpha^{\prime} \uparrow} \\
& \left.-J_{H} \sum_{\alpha \neq \alpha^{\prime}} d_{i \alpha \uparrow}^{\dagger} d_{i \alpha \downarrow} d_{i \alpha^{\prime} \downarrow}^{\dagger} d_{i \alpha^{\prime} \uparrow}\right),
\end{aligned}
$$

where $d_{i \alpha \sigma}^{\dagger}$ denotes the creation operator of the $d$ electron of the magnetic ion on the $t_{2 g}$ orbitals $\alpha=x y(Z), y z(X), z x(Y)$ (in the local axes bound to the oxygen octahedron) with spin $\sigma=\uparrow, \downarrow$. The constants $U_{1}$ and $U_{2}$ denote the Coulomb repulsion among $d$ electrons on the same and on the different $t_{2 g}$ orbitals, respectively, $J_{H}$ denotes the Hund's coupling constant, and $U_{1}=U_{2}+2 J_{H}$, due to the cubic symmetry. The spin-orbit coupling (SOC) is given by

$$
\mathcal{H}_{\mathrm{SOC}}=\lambda \sum_{i} \mathbf{s}_{i} \cdot \mathbf{l}_{i},
$$

where $\mathbf{s}_{i}$ is the spin of the $i$ th electron, and the SOC has been projected into the $t_{2 g}$ manifold, leading to the effective orbital angular momentum $l=1$. Finally, the hopping term $\mathcal{H}_{t}$ has the general form

$$
\begin{aligned}
\mathcal{H}_{t}= & \sum_{i, j} \sum_{\alpha, \beta} \sum_{\sigma} t_{i j, \sigma}^{\alpha \beta} d_{i \alpha \sigma}^{\dagger} d_{j \beta \sigma} \\
& +\widetilde{\sum_{i, j}} \sum_{\alpha, \beta} \sum_{\sigma}\left[\tilde{t}_{i j, \sigma}^{\alpha \beta} d_{i \alpha \sigma}^{\dagger} p_{j \beta \sigma}+\text { H.c. }\right],
\end{aligned}
$$


where the first line gives the direct hopping between magnetic ions, and the second line gives the hopping between magnetic ions and ligand ions, with $p_{j \beta \sigma}$ denoting the annihilation of an electron on the $\beta$ th $p$ orbital of the ligand ion at site $j$. The hopping amplitudes $t_{i j, \sigma}^{\alpha \beta}$ and $\tilde{t}_{i j, \sigma}^{\alpha \beta}$ are determined by the overlaps between the orbitals and are material dependent.

A technical comment is in order here. Usually, the hole picture (in which the magnetic degrees of freedom come from the one-hole states in the $j_{\text {eff }}=1 / 2$ doublets) is used for the description of the magnetic properties of the Kitaev materials, which are in the main focus of this paper. To change all the formulas to the hole picture, one can replace $d_{i \alpha \sigma}^{\dagger}$ with $\tilde{d}_{i \alpha \sigma}$, $d_{i \alpha \sigma}$ with $\tilde{d}_{i \alpha \sigma}^{\dagger}$, and $n_{i \alpha \sigma}$ with $1-n_{i \alpha \sigma}$. With these substitutions, the eigenenergies remain the same (up to the constant energy shift), but we obtain an overall negative sign on each hopping amplitude. Therefore, to keep the above formalism unchanged for the hole picture we simply absorb this negative sign in the hopping parameters. In the following we will omit the tilde of the hole operators for a simpler notation.

A standard superexchange expansion ${ }^{1}$ of the above extended Hubbard model delivers a low-energy effective spin Hamiltonian that, for Kitaev materials such as $\beta-\mathrm{Li}_{2} \mathrm{IrO}_{3}$, can be well described by the nearest-neighbor (NN) $J-K-\Gamma$ model,

$$
\mathcal{H}_{\mathrm{eff}}=\sum_{\langle i j\rangle_{v}} J \mathbf{S}_{i} \cdot \mathbf{S}_{j}+K S_{i}^{\alpha_{\nu}} S_{j}^{\alpha_{\nu}}+\sigma_{\nu} \Gamma\left(S_{i}^{\beta_{\nu}} S_{j}^{\gamma_{\nu}}+S_{i}^{\gamma_{\nu}} S_{j}^{\beta_{v}}\right),
$$

where $\mathbf{S}_{i}$ denotes the pseudospin $j_{\text {eff }}=1 / 2$ operator at site $i$, $\left(\alpha_{\nu}, \beta_{\nu}, \gamma_{v}\right)=(x, y, z),(y, z, x)$, and $(z, x, y)$, respectively, for $v \in\{x, y, z\}$ labeling the three different types of NN Ir-Ir or $\mathrm{Ru}-\mathrm{Ru}$ bonds; the prefactor $\sigma_{\nu}$ equals +1 for two-dimensional materials and can be +1 or -1 (depending on the bond) for the three-dimensional systems $\beta$ - and $\gamma-\mathrm{Li}_{2} \mathrm{IrO}_{3}$. Here $K$ is the Kitaev coupling, $J$ is the Heisenberg coupling and $\Gamma$ is the so-called symmetric exchange anisotropy, which is present in many Kitaev materials [54-59]. These interactions should be thought of as a minimal starting model, as other terms may also be relevant for materials with lower symmetry.

Crucially, the $J, K$, and $\Gamma$ couplings originate from very different microscopic processes. Specifically, as it has been shown in the literature [55,60-63], the Heisenberg interaction $J$ arises from direct virtual hopping processes between $d$ orbitals of magnetic ions, whereas the dominant contribution to the Kitaev interaction $K$ arises from the ligand-mediated hopping. As for $\Gamma$, this arises from a combination of direct and ligand-mediated hopping. As we discuss below, the Raman operator stems from the same underlying microscopic processes as the superexchange Hamiltonian, and each type of these processes gives rise to a different contribution to the Raman response.

\footnotetext{
${ }^{1}$ In case of Kitaev materials, the superexchange expansion usually does not include processes when two holes meet at the same oxygen site in the intermediate state since these intermediate states are higher-energy states and their inclusion only slightly modifies the effective couplings but does not change the picture qualitatively. We note, however, that in systems with small spin-orbit coupling, such as the cuprates, such processes can also lead to small anisotropic interactions [53].
}

\section{MICROSCOPIC DERIVATION OF THE RAMAN OPERATOR}

We first review a number of key steps in the derivation of the Raman operator $\mathcal{R}$ in Mott insulators, with a view on Kitaev materials with strong spin-orbit coupling (SOC). The first step is to write down the total microscopic Hamiltonian,

$$
\mathcal{H}_{\text {tot }}=\mathcal{H}+\mathcal{H}_{\gamma}+\mathcal{H}_{c}
$$

consisting of the extended Hubbard Hamiltonian $\mathcal{H}$, the free photon Hamiltonian

$$
\mathcal{H}_{\gamma}=\sum_{\mathbf{k}, \varepsilon} \omega_{\mathbf{k}} \alpha_{\mathbf{k}, \varepsilon}^{\dagger} \alpha_{\mathbf{k}, \varepsilon}
$$

where $\alpha_{\mathbf{k}, \varepsilon}^{\dagger}$ and $\alpha_{\mathbf{k}, \varepsilon}$ are the creation and destruction operators of a photon with wave vector $\mathbf{k}$ and polarization $\boldsymbol{\varepsilon}$, and $\omega_{\mathbf{k}}$ is the corresponding frequency, and the perturbation $\mathcal{H}_{c}$ that describes the interaction of the electrons (holes) with the electromagnetic (EM) field. The latter arises from the coupling of the light to the electric dipoles induced by the virtual charge transfers between different lattice sites. This coupling can be described by the Peierls substitution, in which a Wilson line operator is attached to the electron (hole) hopping term between magnetic ions as $[1,2,8]$

$$
d_{i \alpha \sigma}^{\dagger} d_{j \beta \sigma} \rightarrow d_{i \alpha \sigma}^{\dagger} d_{j \beta \sigma} e^{\frac{i e}{\hbar c} \int_{\mathbf{r}_{j}}^{\mathbf{r}_{i}} d \mathbf{r} \cdot \mathcal{A}(\mathbf{r})}
$$

(and similarly for the hopping between magnetic and ligand ions), where $\mathcal{A}(\mathbf{r})$ denotes the vector potential of the radiation field. This substitution amounts to replacing $\mathcal{H}_{t}+\mathcal{H}_{c}$ with

$$
\begin{aligned}
\mathcal{H}_{t, \mathcal{A}}= & \sum_{i j} \sum_{\alpha \beta} \sum_{\sigma} t_{i j, \sigma}^{\alpha \beta} d_{i \alpha \sigma}^{\dagger} d_{j \beta \sigma} e^{\frac{i e}{\hbar c} \int_{\mathbf{r}_{j}}^{\mathbf{r}_{i}} d \mathbf{r} \cdot \mathcal{A}(\mathbf{r})} \\
& +\widetilde{\sum_{i j}} \sum_{\alpha \beta} \sum_{\sigma}\left[\tilde{t}_{i j, \sigma}^{\alpha \beta} d_{i \alpha \sigma}^{\dagger} p_{j \beta \sigma} e^{\frac{i e}{h c} \int_{\mathbf{r}_{j}}^{\mathbf{r}_{i}} d \mathbf{r} \cdot \mathcal{A}(\mathbf{r})}+\text { H.c. }\right] .
\end{aligned}
$$

As usual, we consider the case where the wavelengths of the incoming and outgoing photons are much longer than the lattice constant, which allows us to safely replace

$$
\frac{i e}{\hbar c} \int_{\mathbf{r}_{j}}^{\mathbf{r}_{i}} d \mathbf{r} \cdot \mathcal{A}(\mathbf{r}) \simeq \frac{i e}{\hbar c} \mathcal{A} \cdot \delta \mathbf{r}_{i j}, \delta \mathbf{r}_{i j} \equiv \mathbf{r}_{i}-\mathbf{r}_{j},
$$

and then perform an expansion of $\mathcal{H}_{t, \mathcal{A}}$ in powers of the vector potential (which is appropriate for the weak EM fields of Raman experiments), namely

$$
\mathcal{H}_{t, \mathcal{A}}=\mathcal{H}_{t}+\mathcal{H}_{t, \mathcal{A}}^{(1)}+\cdots .
$$

Here $\mathcal{H}_{t}$ is the hopping in the absence of light, and $\mathcal{H}_{t, \mathcal{A}}^{(1)}$ is the leading photon-induced hopping,

$$
\begin{aligned}
\mathcal{H}_{t, \mathcal{A}}^{(1)}= & \sum_{i j} \sum_{\alpha \beta} \sum_{\sigma} t_{i j, \sigma}^{\alpha \beta} d_{i \alpha \sigma}^{\dagger} d_{j \beta \sigma}\left(\frac{i e}{\hbar c} \mathcal{A} \cdot \delta \mathbf{r}_{i j}\right) \\
& +\widetilde{\sum_{i j}} \sum_{\alpha \beta} \sum_{\sigma}\left[\tilde{t}_{i j, \sigma}^{\alpha \beta} d_{i \alpha \sigma}^{\dagger} p_{j \beta \sigma}\left(\frac{i e}{\hbar c} \mathcal{A} \cdot \delta \mathbf{r}_{i j}\right)+\text { H.c. }\right] .
\end{aligned}
$$

We can then express the vector potential in terms of creation and annihilation photon operators, and to that end, it suffices 
to keep only the terms referring to the incoming and outgoing photons, namely,

$$
\begin{aligned}
\mathcal{A} & =g_{\text {in }} \boldsymbol{\varepsilon}_{\text {in }} \alpha_{\mathbf{k}_{\text {in }}, \boldsymbol{\varepsilon}_{\text {in }}} e^{i \mathbf{k}_{\text {in }} \cdot \delta \mathbf{r}_{i j}}+g_{\text {out }} \boldsymbol{\varepsilon}_{\text {out }} \alpha_{\mathbf{k}_{\text {out }}, \boldsymbol{\varepsilon}_{\text {out }}}^{\dagger} e^{i \mathbf{k}_{\text {out }} \delta \delta \mathbf{r}_{i j}} \\
& \simeq g_{\text {in }} \boldsymbol{\varepsilon}_{\text {in }} \alpha_{\mathbf{k}_{\text {in }}, \boldsymbol{\varepsilon}_{\text {in }}}+g_{\text {out }} \boldsymbol{\varepsilon}_{\text {out }} \alpha_{\mathbf{k}_{\text {out }},}^{\dagger}, \boldsymbol{\varepsilon}_{\text {out }} .
\end{aligned}
$$

Here, $\mathbf{k}_{\text {in }}$ and $\boldsymbol{\varepsilon}_{\text {in }}$ (respectively, $\mathbf{k}_{\text {out }}$ and $\boldsymbol{\varepsilon}_{\text {out }}$ ) denote the wave vectors and polarizations of the incoming (respectively, outgoing) photons, and $g_{\text {in }}$ and $g_{\text {out }}$ are constants depending on the photon frequencies $[2,8]$. Furthermore, in the second line we replaced $e^{i \mathbf{k}_{\mathrm{in}} \cdot \delta \mathbf{r}_{i j}} \sim e^{i \mathbf{k}_{\mathrm{out}} \cdot \delta \mathbf{r}_{i j}} \sim 1$, which is accurate in our long-wavelength limit.

Following Refs. $[2,3,8]$, in which the Raman scattering is treated in the framework of the $\mathcal{T}$-matrix formalism, with the photon-induced hopping terms $\mathcal{H}_{t, \mathcal{A}}^{(1)}+\mathcal{H}_{t, \mathcal{A}}^{(2)}+\cdots$ treated as a perturbation, one arrives at the leading contribution to the Raman operator, which is second order in $\mathcal{A}$ (describing a one photon in, one photon out process) $[2,8]$ :

$$
\mathcal{R}=\mathcal{H}_{t, \mathcal{A}}^{(1)} \frac{1}{E-\left(\mathcal{H}+\mathcal{H}_{\gamma}\right)+i \eta} \mathcal{H}_{t, \mathcal{A}}^{(1)},
$$

where $\eta \rightarrow 0^{+}$and $E=2 E_{1 \mathrm{~h}}+\omega_{\text {in }}$ is the eigenenergy of the initial state, in which all magnetic ions have one hole in the $j_{\text {eff }}=1 / 2$ doublet. The next step is to treat $\mathcal{H}_{t}$ as a weak perturbation compared to

$$
\mathcal{H}_{0} \equiv \mathcal{H}_{\text {int }}+\mathcal{H}_{\text {SOC }}+\mathcal{H}_{\text {pd }}+\mathcal{H}_{\gamma}
$$

This allows to expand $\mathcal{R}$ as follows:

$$
\mathcal{R}=\mathcal{H}_{t, \mathcal{A}}^{(1)} \mathcal{G} \sum_{n=0}^{\infty}\left(\mathcal{H}_{t} \mathcal{G}\right)^{n} \mathcal{H}_{t, \mathcal{A}}^{(1)},
$$

where we have defined the resolvent

$$
\mathcal{G}=\left(E-\mathcal{H}_{0}+i \eta\right)^{-1} .
$$

Note that both $\mathcal{H}_{t, \mathcal{A}}^{(1)}$ and $\mathcal{H}_{t}$ include hopping terms on all bonds of the lattice. However, since both the initial and final states belong to the ground-state manifold, only pathways consisting of closed loops contribute to the Raman operator.

Incidentally, replacing $\mathcal{H}_{t, \mathcal{A}}^{(1)}$ by $\mathcal{H}_{t}$ in Eq. (16) gives the leading contributions to the effective spin Hamiltonian $\mathcal{H}_{\text {eff }}$. More specifically,

$$
\mathcal{H}_{\text {eff }}=\mathcal{H}_{t} \mathcal{G} \sum_{n=0}^{\infty}\left(\mathcal{H}_{t} \mathcal{G}\right)^{n} \mathcal{H}_{t}+\text { other terms },
$$

where the other terms in the formal expansion [64] of $\mathcal{H}_{\text {eff }}$ can be safely disregarded for our purposes.

\section{RAMAN OPERATOR IN KITAEV MATERIALS}

We are now ready to apply the above general formalism to the case of the Kitaev materials and highlight the main new insights of this work. In particular, we will explicitly demonstrate that despite the fact that the microscopic processes underlying the Raman operator and the superexchange Hamiltonian are very similar in the Kitaev materials $[55,60,61,63]$, the presence of multiple nonequivalent superexchange paths contributing to the coupling between the magnetic moments on a given bond leads to the contributions to the Raman operator that goes beyond the Loudon-Fleury theory [35].

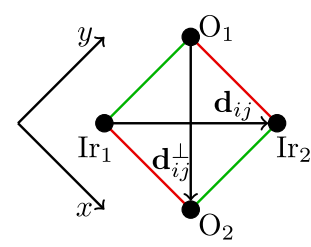

FIG. 1. The square plaquette that is relevant for the superexchange processes between two magnetic ions sharing a $z$ bond in $\mathrm{A}_{2} \mathrm{IrO}_{3}$ compounds (e.g., $\beta$ - $\mathrm{Li}_{2} \mathrm{IrO}_{3}$ ). The same plaquette provides the superexchange processes in $\alpha-\mathrm{RuCl}_{3}$ with substitution $\mathrm{Ir} \rightarrow \mathrm{Ru}$, $\mathrm{O} \rightarrow \mathrm{Cl}$.

Recall that in the Loudon-Fleury theory, the contribution $\mathcal{R}_{i j}$ to the total Raman operator from a given bond $(i j)$, is simply given by the superexchange interactions $\mathcal{H}_{\mathrm{eff}, i j}$ on that bond, weighted by a bond-specific polarization-dependent factor. However, in systems with multiple nonequivalent superexchange paths, the polarization factors that come from the operators $\mathcal{H}_{t, \mathcal{A}}^{(1)}$ appearing at the first and last steps of the perturbative expansion of Eq. (16) give unequal weights to different paths. Hence, the summation over these paths leads to a Raman operator $\mathcal{R}_{i j}$ (on the given bond), which is, in general, not proportional to $\mathcal{H}_{\text {eff }, i j}$ (obtained by summing up the contributions from all possible paths with equal weight).

Let us now begin with reexamining the various superexchange paths contributing to the effective spin Hamiltonian of the Kitaev materials. In all of them, the local environment of the magnetic ions is that of an octahedron ligand cage, see, e.g., Fig. 5 for the case of $\beta$ - $\mathrm{Li}_{2} \mathrm{IrO}_{3}$. The virtual hopping processes leading to the $J-K-\Gamma$ model (and the ones contributing to the Raman operator) are confined to a plaquette consisting of two magnetic ions and two ligand ions. For iridium Kitaev materials, for example, the plaquette is formed by two iridium and two oxygen ions (see, e.g., Fig. 1 for the case of two $\mathrm{Ir}^{4+}$ ions sharing a $z$ bond in $\beta$ - $\mathrm{Li}_{2} \mathrm{IrO}_{3}$ ), while for $\alpha-\mathrm{RuCl}_{3}$ it is formed by two ruthenium and two chlorine ions. For concreteness, in the following discussion we will use notations for the iridates, but the final results will be exactly the same for $\alpha-\mathrm{RuCl}_{3}$ as well.

We will carry out our analysis for a $z$ bond formed by two iridium ions, $\operatorname{Ir}_{1}$ and $\operatorname{Ir}_{2}$. The results for other types of bonds can be obtained in a similar way (or simply by symmetry, if present). Using the frame of Fig. 1, the vector connecting these two ions is $\mathbf{d}_{i j}=\mathbf{x}+\mathbf{y}$ (in appropriate length units), while the vector connecting the two oxygen sites, $\mathrm{O}_{1}$ and $\mathrm{O}_{2}$, is $\mathbf{d}_{i j}^{\perp}=\mathbf{x}-\mathbf{y}$. Finally, the vectors connecting $\operatorname{Ir}_{1}$ with $\mathrm{O}_{1}$ and $\mathrm{O}_{2}$ are, respectively, $\mathbf{y}$ and $\mathbf{x}$. The hopping matrix elements corresponding to this $z$ bond are listed in Table I.

TABLE I. The matrix elements of $\mathcal{H}_{t}$ (in the hole picture) related to the $z$ bond for $\beta-\mathrm{Li}_{2} \mathrm{IrO}_{3}$. All matrix elements are real.

\begin{tabular}{lc}
\hline transfer path & hopping amplitude \\
\hline $\operatorname{Ir}[x z(Y)$ or $y z(X)] \rightarrow \mathrm{O}\left(p_{z}\right)$ & $t$ \\
$\operatorname{Ir}_{1}[x z(Y)] \rightarrow \operatorname{Ir}_{2}[x z(Y)]$ & $t_{1}$ \\
$\operatorname{Ir}_{1}[y z(X)] \rightarrow \operatorname{Ir}_{2}[y z(X)]$ & $t_{1}$ \\
$\operatorname{Ir}_{1}[x y(Z)] \rightarrow \operatorname{Ir}_{2}[x y(Z)]$ & $t_{3}$ \\
\hline \hline
\end{tabular}




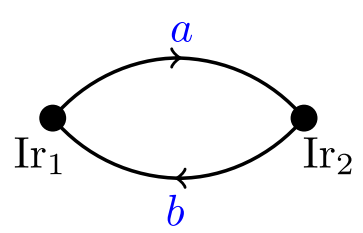

FIG. 2. Direct hopping ( $a-b$ and $b-a)$.

There are three different types of paths on the plaquette of Fig. 1: (i) direct hopping (Fig. 2), (ii) oxygen-mediated hopping (Fig. 3), and (iii) mixed hopping (Fig. 4). The direct hopping contributes at the lowest order of the perturbation $[n=0$ in Eq. (16)], the oxygen-mediated hopping processes arise at fourth order $(n=2)$, and the mixed direct/oxygenmediated hopping processes arise at third order $(n=1)$. Mathematically, the corresponding amplitudes for each of these types of processes can be obtained by performing a spectral decomposition of the resolvent $\mathcal{G}$ of Eq. (17) in terms of the relevant virtual excitations. These include the intermediate two-hole states on iridium sites, $\left|D_{\mu}\right\rangle$, where $\mu=$ $1,2, \ldots, 15$, obtained by the diagonalization of $\mathcal{H}_{\text {int }}[62,63]$ (see also Table IV in Appendix 1), and the intermediate onehole states on oxygen sites, $\left|O_{v}\right\rangle$, where $v=1,2$ labels one of the two oxygen ions:

$$
\begin{aligned}
\mathcal{G} & =\sum_{\mu=1}^{15} \frac{\left|D_{\mu}\right\rangle\left\langle D_{\mu}\right|}{\left(2 E_{1 \mathrm{~h}}-E_{2 \mathrm{~h}}-E_{0 \mathrm{~h}}\right)+\omega_{\mathrm{in}}+i \eta}+\sum_{\nu=1}^{2} \frac{\left|O_{\nu}\right\rangle\left\langle O_{\nu}\right|}{\omega_{\mathrm{in}}-\Delta_{\mathrm{pd}}+i \eta} \\
& \equiv \mathcal{G}_{D}+\mathcal{G}_{O} .
\end{aligned}
$$

Here $\omega_{\text {in }}$ is the incoming photon frequency, $\Delta_{\text {pd }}$ is the charge transfer energy between $\mathrm{Ir}^{4+}$ and $\mathrm{O}^{2-}$, and we have also defined $E_{0 \mathrm{~h}}, E_{1 \mathrm{~h}}$, and $E_{2 \mathrm{~h}}$ to be the zero-, one-, and two-hole eigenenergies.

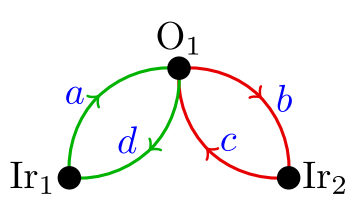

(a) path $1(a-b-c-d)$ and 2 $(c-d-a-b)$

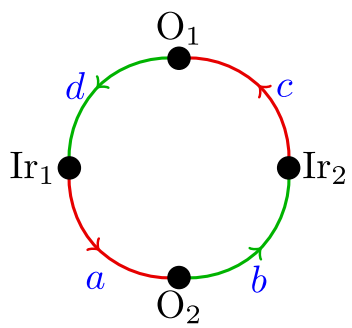

(c) path $5(a-b-c-d)$ and 6 $(c-d-a-b)$

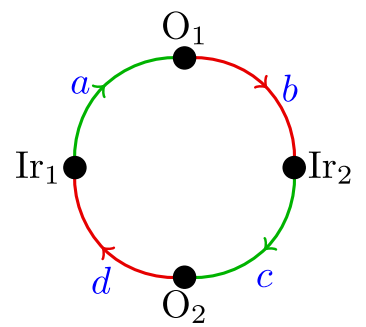

(b) path $3(a-b-c-d)$ and 4 $(c-d-a-b)$

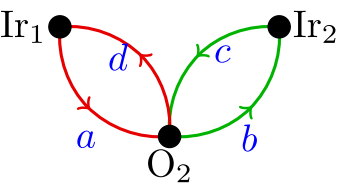

(d) path $7(a-b-c-d)$ and 8 $(c-d-a-b)$
FIG. 3. The eight different oxygen-mediated hopping paths connecting $\operatorname{Ir}_{1}$ and $\operatorname{Ir}_{2}$ in the plaquette of Fig. 1.

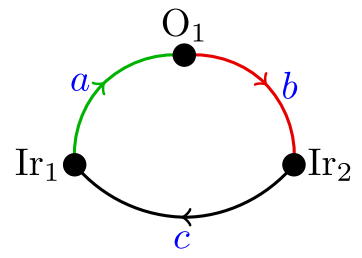

(a) path $1(a-b-c)$ and $2(c-a-b)$

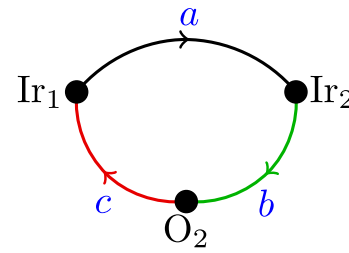

(c) path $5(a-b-c)$ and $6(c-a-b)$

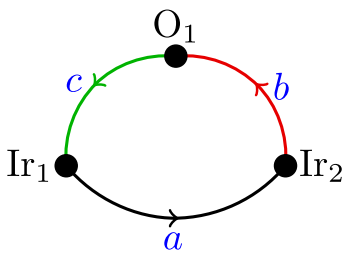

(b) path $3(a-b-c)$ and $4(c-a-b)$

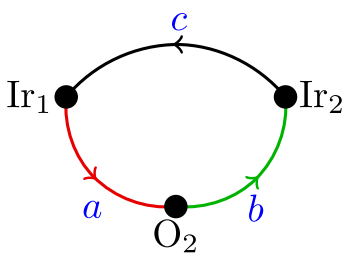

(d) path $7(a-b-c)$ and $8(c-a-b)$
FIG. 4. The eight different mixed hopping paths connecting $\operatorname{Ir}_{1}$ and $\mathrm{Ir}_{2}$ in the basic plaquette of Fig. 1.

The effective Raman operator lives in the low-energy sector with magnetic ions being in their $j_{\text {eff }}=1 / 2$ ground state manifold. For two neighboring Ir ions, this space is spanned by the four configurations written in the $\left|j_{\text {eff }, 1}^{z}, j_{\text {eff, } 2}^{z}\right\rangle$ representation:

$$
\begin{aligned}
& \left\{\left|\psi_{1}\right\rangle,\left|\psi_{2}\right\rangle,\left|\psi_{3}\right\rangle,\left|\psi_{4}\right\rangle\right\} \\
& \quad \equiv\left\{\left|\frac{1}{2}, \frac{1}{2}\right\rangle,\left|\frac{1}{2},-\frac{1}{2}\right\rangle,\left|-\frac{1}{2}, \frac{1}{2}\right\rangle,\left|-\frac{1}{2},-\frac{1}{2}\right\rangle\right\} .
\end{aligned}
$$

One then evaluates the matrix elements $\left\langle\psi_{n}|\mathcal{R}| \psi_{n^{\prime}}\right\rangle$ of the Raman operator in this $4 \times 4$ basis, and then expresses the resulting matrix in terms of the pseudospin operators $\mathbf{S}_{i}$ and $\mathbf{S}_{j}$ to obtain the effective spin representation of $\mathcal{R}$.

For what follows, it is also expedient to define the following generic polarization factors that arise from the coupling of the incoming and outgoing photon to, respectively, the first and last bond of the virtual hopping paths involved in Eq. (16) as

$$
\begin{aligned}
P_{d d} & \equiv \zeta\left(\varepsilon_{\text {in }} \cdot \mathbf{d}_{i j}\right)\left(\varepsilon_{\text {out }} \cdot \mathbf{d}_{i j}\right), \\
P_{d^{\perp} d^{\perp}} & \equiv \zeta\left(\varepsilon_{\text {in }} \cdot \mathbf{d}_{i j}^{\perp}\right)\left(\varepsilon_{\text {out }} \cdot \mathbf{d}_{i j}^{\perp}\right), \\
P_{d d^{\perp}} & \equiv \zeta\left(\varepsilon_{\text {in }} \cdot \mathbf{d}_{i j}\right)\left(\boldsymbol{\varepsilon}_{\text {out }} \cdot \mathbf{d}_{i j}^{\perp}\right), \\
P_{d^{\perp} d} & \equiv \zeta\left(\varepsilon_{\text {in }} \cdot \mathbf{d}_{i j}^{\perp}\right)\left(\boldsymbol{\varepsilon}_{\text {out }} \cdot \mathbf{d}_{i j}\right),
\end{aligned}
$$

where $\zeta=-\frac{e^{2}}{\hbar^{2} c^{2}} g_{\text {in }} g_{\text {out }}$.

\section{A. Raman operator from direct hopping}

There are two direct hopping processes (see Fig. 2) connecting the two $\mathrm{Ir}^{4+}$ ions of the basic plaquette of Fig. 1. Since these processes are of second order in the hopping [i.e., $n=0$ in Eq. (16)] and do not involve the oxygen sites, the corresponding Raman operator is given by $\mathcal{R}^{\mathrm{dir}}=\mathcal{H}_{t, \mathcal{A}}^{(1)} \mathcal{G}_{D} \mathcal{H}_{t, \mathcal{A}}^{(1)}$, or, equivalently,

$$
\mathcal{R}^{\mathrm{dir}}=\sum_{\mu} \frac{\mathcal{H}_{t, \mathcal{A}}^{(1)}\left|D_{\mu}\right\rangle\left\langle D_{\mu}\right| \mathcal{H}_{t, \mathcal{A}}^{(1)}}{\left(2 E_{1 \mathrm{~h}}-E_{2 \mathrm{~h}}-E_{0 \mathrm{~h}}\right)+\omega_{\mathrm{in}}+i \eta} .
$$

The details of the computation of the matrix elements of $\mathcal{R}^{\mathrm{dir}}$ are provided in Appendix 1. The resulting expression for $\mathcal{R}_{i j}^{\mathrm{dir}}$ 
TABLE II. Polarization factors for the eight oxygen-mediated hopping paths of Fig. 3 , and $P_{d d}, P_{d^{\perp} d^{\perp}}, P_{d d^{\perp}}$, and $P_{d^{\perp} d}$ are defined in Eq. (20).

\begin{tabular}{lc}
\hline \hline path $\ell$ & polarization factor $p_{\ell}^{O}$ \\
\hline 1 & $\zeta\left(\varepsilon_{\text {in }} \cdot \mathbf{y}\right)\left[\varepsilon_{\text {out }} \cdot(-\mathbf{y})\right]=-\left(P_{d d}+P_{d^{\perp} d^{\perp}}-P_{d d^{\perp}}-P_{d^{\perp} d}\right) / 4$ \\
2 & $\zeta\left[\varepsilon_{\text {in }} \cdot(-\mathbf{x})\right]\left(\varepsilon_{\text {out }} \cdot \mathbf{x}\right)=-\left(P_{d d}+P_{d^{\perp} d^{\perp}}+P_{d d^{\perp}}+P_{d^{\perp} d}\right) / 4$ \\
3 & $\zeta\left(\varepsilon_{\text {in }} \cdot \mathbf{y}\right)\left[\varepsilon_{\text {out }} \cdot(-\mathbf{x})\right]=-\left(P_{d d}-P_{d^{\perp} d^{\perp}}+P_{d d^{\perp}}-P_{d^{\perp} d}\right) / 4$ \\
4 & $\zeta\left[\varepsilon_{\text {in }} \cdot(-\mathbf{y})\right]\left(\varepsilon_{\text {out }} \cdot \mathbf{x}\right)=-\left(P_{d d}-P_{d^{\perp} d^{\perp}}+P_{d d^{\perp}}-P_{d^{\perp} d}\right) / 4$ \\
5 & $\zeta\left(\varepsilon_{\text {in }} \cdot \mathbf{x}\right)\left[\varepsilon_{\text {out }} \cdot(-\mathbf{y})\right]=-\left(P_{d d}-P_{d^{\perp} d^{\perp}}-P_{d d^{\perp}}+P_{d^{\perp} d}\right) / 4$ \\
6 & $\zeta\left[\varepsilon_{\text {in }} \cdot(-\mathbf{x})\right]\left(\varepsilon_{\text {out }} \cdot \mathbf{y}\right)=-\left(P_{d d}-P_{d^{\perp} d^{\perp}}-P_{d d^{\perp}}+P_{d^{\perp} d}\right) / 4$ \\
7 & $\zeta\left(\varepsilon_{\text {in }} \cdot \mathbf{x}\right)\left[\varepsilon_{\text {out }} \cdot(-\mathbf{x})\right]=-\left(P_{d d}+P_{d^{\perp} d^{\perp}}+P_{d d^{\perp}}+P_{d^{\perp} d}\right) / 4$ \\
8 & $\zeta\left[\varepsilon_{\text {in }} \cdot(-\mathbf{y})\right]\left(\varepsilon_{\text {out }} \cdot \mathbf{y}\right)=-\left(P_{d d}+P_{d^{\perp} d^{\perp}}-P_{d d^{\perp}}-P_{d^{\perp} d}\right) / 4$ \\
\hline \hline
\end{tabular}

on the bond $\langle i j\rangle_{z}$ in terms of spin operators is

$$
\mathcal{R}_{\langle i j\rangle_{z}}^{\mathrm{dir}}=-P_{d d}\left(J^{(2)} \mathbf{S}_{i} \cdot \mathbf{S}_{j}+K^{(2)} S_{i}^{z} S_{j}^{z}\right),
$$

where $K^{(2)}$ and $J^{(2)}$ are coupling constants (explicit analytic expressions of them are given in Appendix 4, and we will comment on their numerical values for the case of $\beta-\mathrm{Li}_{2} \mathrm{IrO}_{3}$ in Sec. VI), and the superscript (2) specifies that they are obtained in second-order perturbation theory. We should note here that $K^{(2)}$ and $J^{(2)}$ depend on the frequency $\omega_{\text {in }}$ of the incoming light, but in the limit $\omega_{\text {in }} \rightarrow 0$, they reduce to the second-order contributions to the effective couplings $K$ and $J$ of the effective $J-K-\Gamma$ model, as they arise from the same microscopic processes. Indeed, starting from Eq. (18) one can show that, for $\omega_{\text {in }} \rightarrow 0$, the spin terms inside the bracket of Eq. (22) are precisely the contributions to $\mathcal{H}_{\text {eff, }}\langle i j\rangle_{z}$ from direct hopping, namely,

$$
\omega_{\text {in }} \mapsto 0: \mathcal{R}_{\langle i j\rangle_{z}}^{\mathrm{dir}}=-P_{d d} \mathcal{H}_{\text {eff },\langle i j\rangle_{z}}^{\mathrm{dir}} .
$$

Hence, the leading Raman operator coming from direct hopping processes has a Loudon-Fleury form.

\section{B. Raman operator from oxygen-mediated hopping}

Turning to oxygen-mediated hopping processes, their leading contribution to the Raman operator appears at fourth order in the hopping $[n=2$ in Eq. (16)] and has the form

$$
\mathcal{R}^{\text {med }}=\mathcal{H}_{t, \mathcal{A}}^{(1)} \mathcal{G}_{O} \mathcal{H}_{t} \mathcal{G}_{D} \mathcal{H}_{t} \mathcal{G}_{O} \mathcal{H}_{t, \mathcal{A}}^{(1)} \text {. }
$$

In total, for the bond $\langle i j\rangle_{z}$, there are eight different paths contributing to this operator, labeled by $\ell=1, \ldots 8$, four of which begin from $\operatorname{Ir}_{1}$ and the other four from $\operatorname{Ir}_{2}$, see Fig. 3 . Each path $\ell$ gives rise to a polarization factor $p_{\ell}^{O}$ (provided in Table II) multiplying an effective spin operator $\mathcal{H}_{\ell}^{O}$, namely,

$$
\mathcal{R}_{\langle i j\rangle_{z}}^{\mathrm{med}}=\sum_{\ell=1}^{8} p_{\ell}^{O} \mathcal{H}_{\ell}^{O}
$$

(see details in Appendix 2). The final form of the Raman operator from oxygen-mediated hopping is given by

$$
\mathcal{R}_{\langle i j\rangle_{z}}^{\mathrm{med}}=-\frac{P_{d d}}{4} K^{(4)} S_{i}^{z} S_{j}^{z}-\frac{P_{d^{\perp} d^{\perp}}}{4}\left(J^{\prime(4)} \mathbf{S}_{i} \cdot \mathbf{S}_{j}+K^{\prime(4)} S_{i}^{z} S_{j}^{z}\right),
$$

where the frequency-dependent constants $K^{(4)}, J^{\prime(4)}$, and $K^{\prime(4)}$ can be obtained numerically for the convenience of
TABLE III. Polarization factors of the eight mixed hopping paths of Fig. 4. For the definitions of $P_{d d}, P_{d^{\perp} d^{\perp}}, P_{d d^{\perp}}$, and $P_{d^{\perp} d}$ see Eq. (20).

\begin{tabular}{lc}
\hline \hline path $\ell$ & polarization factor $p_{\ell}^{m}$ \\
\hline 1 & $\zeta\left(\boldsymbol{\varepsilon}_{\text {in }} \cdot \mathbf{y}\right)\left[\boldsymbol{\varepsilon}_{\text {out }} \cdot\left(-\mathbf{d}_{i j}\right)\right]=-\left(P_{d d}-P_{d^{\perp}}\right) / 2$ \\
2 & $\zeta\left[\varepsilon_{\text {in }} \cdot\left(-\mathbf{d}_{i j}\right)\right]\left(\boldsymbol{\varepsilon}_{\text {out }} \cdot \mathbf{x}\right)=-\left(P_{d d}+P_{d d^{\perp}}\right) / 2$ \\
3 & $\zeta\left(\varepsilon_{\text {in }} \cdot \mathbf{d}_{i j}\right)\left[\boldsymbol{\varepsilon}_{\text {out }} \cdot(-\mathbf{y})\right]=-\left(P_{d d}-P_{d d^{\perp}}\right) / 2$ \\
4 & $\zeta\left[\varepsilon_{\text {in }} \cdot(-\mathbf{x})\right]\left(\boldsymbol{\varepsilon}_{\text {out }} \cdot \mathbf{d}_{i j}\right)=-\left(P_{d d}+P_{d^{\perp}}\right) / 2$ \\
5 & $\zeta\left(\varepsilon_{\text {in }} \cdot \mathbf{d}_{i j}\right)\left[\boldsymbol{\varepsilon}_{\text {out }} \cdot(-\mathbf{x})\right]=-\left(P_{d d}+P_{d d^{\perp}}\right) / 2$ \\
6 & $\zeta\left[\varepsilon_{\text {in }} \cdot(-\mathbf{y})\right]\left(\boldsymbol{\varepsilon}_{\text {out }} \cdot \mathbf{d}_{i j}\right)=-\left(P_{d d}-P_{d^{\perp} d}\right) / 2$ \\
7 & $\zeta\left(\varepsilon_{\text {in }} \cdot \mathbf{x}\right)\left[\boldsymbol{\varepsilon}_{\text {out }} \cdot\left(-\mathbf{d}_{i j}\right)\right]=-\left(P_{d d}+P_{d^{\perp}}\right) / 2$ \\
8 & $\zeta\left[\boldsymbol{\varepsilon}_{\text {in }} \cdot\left(-\mathbf{d}_{i j}\right)\right]\left(\boldsymbol{\varepsilon}_{\text {out }} \cdot \mathbf{y}\right)=-\left(P_{d d}-P_{d d^{\perp}}\right) / 2$ \\
\hline \hline
\end{tabular}

calculation (see Appendix 4 for their analytic expressions). Comparing with the corresponding contributions to the effective spin Hamiltonian (computed with $\omega_{\text {in }}=0$ ),

$$
\mathcal{H}_{\mathrm{eff},\langle i j\rangle_{z}}^{\mathrm{med}}=\sum_{\ell=1}^{8} \mathcal{H}_{\ell}^{O}=K S_{i}^{z} S_{j}^{z}
$$

shows that the Raman operator from oxygen-mediated hopping does not take a Loudon-Fleury form, i.e., $\mathcal{R}_{\langle i j\rangle_{z}}^{\mathrm{med}}$ computed on a given bond is not proportional to $\mathcal{H}_{\mathrm{eff},\langle i j\rangle_{z}}^{\text {med }}$.

\section{Raman operator from mixed hopping}

Let us now discuss virtual processes that involve both direct and oxygen-mediated hopping. The leading contributions to the corresponding Raman operator appear first at third order in the hopping [ $n=1$ in Eq. (16)], and take the form

$$
\mathcal{R}^{\text {mix }}=\mathcal{H}_{t, \mathcal{A}}^{(1)} \mathcal{G H}_{t} \mathcal{G H}_{t, \mathcal{A}}^{(1)} \text {. }
$$

In total, for the bond $\langle i j\rangle_{z}$, there are again eight different paths contributing to this operator (see Fig. 4), labeled by $\ell=1-8$. As in the case of oxygen-mediated hopping, here too, each path $\ell$ gives rise to a polarization factor (provided in Table III) multiplying an effective spin operator $\mathcal{H}_{\ell}^{\mathrm{m}}$, namely,

$$
\mathcal{R}_{\langle i j\rangle_{z}}^{\mathrm{mix}}=\sum_{\ell=1}^{8} p_{\ell}^{m} \mathcal{H}_{\ell}^{\mathrm{m}}
$$

(see details in Appendix 3), which takes the form

$$
\mathcal{R}_{\langle i j\rangle_{z}}^{\mathrm{mix}}=-\frac{P_{d d}}{2} \Gamma^{(3)}\left(S_{i}^{x} S_{j}^{y}+S_{i}^{y} S_{j}^{x}\right)-\frac{P_{d^{\perp} d}-P_{d d^{\perp}}}{2} i h_{\Gamma}^{(3)}\left(S_{i}^{z}+S_{j}^{z}\right),
$$

where the frequency-dependent constants $\Gamma^{(3)}$ and $h_{\Gamma}^{(3)}$ are determined numerically again (see Appendix 4 for their analytic expressions), and the additional factor $i$ in front of the real parameter $h_{\Gamma}^{(3)}$ ensures time-reversal symmetry. Comparing again with the corresponding contributions to the effective spin Hamiltonian (computed with $\omega_{\text {in }}=0$ ),

$$
\mathcal{H}_{\text {eff, }\{i j\rangle_{z}}^{\text {mix }}=\sum_{\ell=1}^{8} \mathcal{H}_{\ell}^{\mathrm{m}}=\Gamma\left(S_{i}^{x} S_{j}^{y}+S_{i}^{y} S_{j}^{x}\right),
$$

we see that, as in the case of oxygen-mediated hopping, the Raman operator from mixed processes does not take a 
Loudon-Fleury form, i.e., $\mathcal{R}_{\langle i j\rangle_{z}}^{\operatorname{mix}}$ is not proportional to $\mathcal{H}_{\mathrm{eff},\langle i j\rangle_{z}}^{\mathrm{mix}}$. In particular, the extra, non-Loudon-Fleury term [second term in Eq. (30)] takes the form of an effective, local magnetic field term $\propto h_{\Gamma}^{(3)}$, along the quantization axis $z$ associated with the $\mathrm{Ir}_{1}-\mathrm{Ir}_{2}$ bond of Fig. 1 .

\section{Total Raman operator}

Collecting the various contributions to the Raman operator on $z$ bonds, and the analogous expressions for $x$ bonds and $y$ bonds gives the total Raman operator

$$
\begin{aligned}
\mathcal{R}= & \sum_{\langle i j\rangle_{v}}\left\{\mathcal{P}_{i j, J} \mathbf{S}_{i} \cdot \mathbf{S}_{j}+\mathcal{P}_{i j, K} S_{i}^{\alpha_{\nu}} S_{j}^{\alpha_{\nu}}+\mathcal{P}_{i j, \Gamma}\left(S_{i}^{\beta_{\nu}} S_{j}^{\gamma_{\nu}}+S_{i}^{\gamma_{\nu}} S_{j}^{\beta_{\nu}}\right)\right. \\
& \left.+\mathcal{P}_{i j, h_{\Gamma}} S\left(S_{i}^{\alpha_{\nu}}+S_{j}^{\alpha_{\nu}}\right)\right\},
\end{aligned}
$$

where notations for $\left(\alpha_{v}, \beta_{v}, \gamma_{v}\right)$ are the same as in Eq. (5) and

$$
\begin{aligned}
\mathcal{P}_{i j, J} & \equiv-P_{d d} J^{(2)}-\frac{1}{4} P_{d^{\perp} d^{\perp} J^{\prime(4)}} \\
\mathcal{P}_{i j, K} & \equiv-P_{d d}\left(K^{(2)}+\frac{1}{4} K^{(4)}\right)-\frac{1}{4} P_{d^{\perp} d^{\perp}} K^{(4)}, \\
\mathcal{P}_{i j, \Gamma} & \equiv-\frac{1}{2} P_{d d} \Gamma^{(3)}, \\
\mathcal{P}_{i j, h_{\Gamma}} & \equiv-\frac{1}{2}\left(P_{d^{\perp} d}-P_{d d^{\perp}}\right) i h_{\Gamma}^{(3)} / S .
\end{aligned}
$$

We repeat here that the various constants entering the Raman operator, i.e., $J^{(2)}, J^{\prime(4)}, K^{(2)}, K^{(4)}, K^{\prime(4)}, \Gamma^{(3)}$, and $h_{\Gamma}^{(3)}$, are frequency dependent and are not directly related to the effective couplings in the original superexchange Hamiltonian (5). However, the following relations hold, up to fourth order in $\mathcal{H}_{t}$,

$$
\omega_{\text {in }} \rightarrow 0: J^{(2)}=J, K^{(2)}+K^{(4)}=K, \quad \Gamma^{(3)}=\Gamma .
$$

Note further that the constants $J^{\prime(4)}, K^{\prime(4)}$, and $h_{\Gamma}^{(3)}$ do not appear in the effective spin Hamiltonian and are the ones that are responsible for the non-Loudon-Fleury Raman scattering.

\section{BOSONIC REPRESENTATION OF $\mathcal{R}$ IN MAGNETICALLY ORDERED STATES}

Having established the leading contributions to the Raman operator, we can now turn to its magnon representation in the low-temperature, magnetically ordered states of iridates, such as $\beta-\mathrm{Li}_{2} \mathrm{IrO}_{3}$. To describe the magnon excitations above an ordered state we first need to relabel the positions of the spins $i \rightarrow(\mathbf{R}, \mu)$, where $\mathbf{R}$ is the position of the magnetic unit cell, and $\mu$ labels the different spin sublattices in the given magnetic state around which we wish to perform the $1 / S$ semiclassical expansion. The relabeling allows for the substitutions

$$
\mathbf{S}_{i} \rightarrow \mathbf{S}_{\mathbf{R}, \mu}, \mathbf{S}_{j} \rightarrow \mathbf{S}_{\mathbf{R}+\mathbf{t}_{\mu \mu^{\prime}}, \mu^{\prime}}, \mathcal{P}_{i j, I} \rightarrow \mathcal{P}_{\mu \mu^{\prime}, I}, \sum_{\langle i j\rangle_{v}} \rightarrow \frac{1}{2} \sum_{\mathbf{R},\left(\mu \mu^{\prime}\right)_{v}}
$$

where $\mathbf{t}_{\mu \mu^{\prime}}$ is a primitive translation of the magnetic superlattice that connects the sites $i$ and $j$. Next, we rotate the spin operators from the global laboratory frame to local reference frames

$$
\widetilde{\mathbf{S}}_{\mathbf{R}, \mu}=\mathbf{U}_{\mu} \cdot \mathbf{S}_{\mathbf{R}, \mu}
$$

where $\mathbf{U}_{\mu}$ is a rotation matrix, which depends on the direction of the $\mu$ th spin sublattice in the classical configuration, and express the operators $\widetilde{\mathbf{S}}_{\mathbf{R}, \mu}$ in terms of bosonic operators $a_{\mathbf{R}, \mu}$ via the standard Holstein-Primakoff expansion (to leading order),

$$
\begin{aligned}
& \tilde{S}_{\mathbf{R}, \mu}^{x} \simeq \sqrt{S / 2}\left(a_{\mathbf{R}, \mu}^{\dagger}+a_{\mathbf{R}, \mu}\right), \\
& \tilde{S}_{\mathbf{R}, \mu}^{y} \simeq-i \sqrt{S / 2}\left(a_{\mathbf{R}, \mu}-a_{\mathbf{R}, \mu}^{\dagger}\right), \\
& \widetilde{S}_{\mathbf{R}, \mu}^{z}=S-a_{\mathbf{R}, \mu}^{\dagger} a_{\mathbf{R}, \mu} .
\end{aligned}
$$

Replacing in Eq. (32) and expanding in powers of $1 / \sqrt{S}$ gives

$$
\mathcal{R}=\mathcal{R}_{0}+\mathcal{R}_{1}+\mathcal{R}_{2}+\mathcal{O}\left(S^{1 / 2}\right)
$$

where $\mathcal{R}_{0}$ corresponds to a constant term and does not contribute to the scattering, whereas $\mathcal{R}_{1}$ and $\mathcal{R}_{2}$ describe, respectively, one-magnon and two-magnon scattering.

Knowing the Raman operator, we can then compute the Raman intensity as

$$
\mathcal{I}(\Omega)=\frac{1}{2 \pi} \int_{-\infty}^{\infty} d t e^{i \Omega t}\langle\mathcal{R}(t) \mathcal{R}(0)\rangle
$$

where $\Omega=\omega_{\text {in }}-\omega_{\text {out }}$ is the total energy transferred to the system (in units of $\hbar=1$ ) and $\langle\cdots\rangle$ denotes the ground state average. In the following, we will assume that $\Omega \ll \omega_{\text {in, out }}$.

\section{A. One-magnon scattering}

The one-magnon Raman operator in Eq. (37) reads

$$
\mathcal{R}_{1}=\sum_{\mathbf{R},\left(\mu \mu^{\prime}\right)_{v}}\left\{V_{\mu \mu^{\prime}}^{(1)}\left(a_{\mathbf{R}, \mu}+a_{\mathbf{R}+\mathbf{t}_{\mu \mu^{\prime}}, \mu^{\prime}}\right)+\text { H.c. }\right\},
$$

where

$$
\begin{aligned}
V_{\mu \mu^{\prime}}^{(1)}= & \frac{S^{3 / 2}}{2 \sqrt{2}}\left\{\mathcal{P}_{\mu \mu^{\prime}, J}\left[\mathbf{U}_{\mu} \cdot \mathbf{U}_{\mu^{\prime}}^{-1}\right]_{x-i y, z}\right. \\
& +\mathcal{P}_{\mu \mu^{\prime}, K}\left[\mathbf{U}_{\mu}\right]_{x-i y, \alpha_{\nu}}\left[\mathbf{U}_{\mu^{\prime}}^{-1}\right]_{\alpha_{\nu} z} \\
& +\mathcal{P}_{\mu \mu^{\prime}, \Gamma}\left(\left[\mathbf{U}_{\mu}\right]_{x-i y, \beta_{\nu}}\left[\mathbf{U}_{\mu^{\prime}}^{-1}\right]_{\gamma_{v} z}+\left[\mathbf{U}_{\mu}\right]_{x-i y, \gamma_{\nu}}\left[\mathbf{U}_{\mu^{\prime}}^{-1}\right]_{\beta_{\nu} z}\right) \\
& \left.+\mathcal{P}_{\mu \mu^{\prime}, h_{\Gamma}}\left[\mathbf{U}_{\mu}^{-1}\right]_{\alpha_{\nu}, x-i y}\right\},
\end{aligned}
$$

where we use the notation $[\cdots]_{x-i y, \alpha} \equiv[\cdots]_{x \alpha}-i[\cdots]_{y \alpha}$, $\left(\alpha_{v}, \beta_{\nu}, \gamma_{\nu}\right)$ follows the same definition given after Eq. (5), and $x, y, z$ in the subscript indicate the first, the second, and the third component of the matrix, respectively (e.g. $[\ldots]_{x y}$ denotes the element from the first row and the second column of the matrix $[\ldots])$.

Next we switch to momentum space via Fourier transform,

$$
a_{\mathbf{R}, \mu}=\frac{1}{\sqrt{\mathcal{N} / \mathcal{N}_{m}}} \sum_{\mathbf{q}} e^{i \mathbf{q} \cdot\left(\mathbf{R}+\boldsymbol{\rho}_{\mu}\right)} a_{\mu, \mathbf{q}}
$$

where $\mathcal{N}$ is the total number of sites, $\mathcal{N}_{m}$ is the number of sites inside the magnetic unit cell, and $\rho_{\mu}$ denotes the position of the $\mu$ th sublattice inside the unit cell. Keeping only the $\mathbf{q}=0$ components, we can write

$$
\mathcal{R}_{1, \mathbf{q}=0}=\mathbf{V}^{(1)} \cdot \mathbf{x}_{\mathbf{q}=\mathbf{0}},
$$

where $\mathbf{x}_{\mathbf{q}}=\left(a_{1, \mathbf{q}}, \ldots, a_{\mathcal{N}_{m}, \mathbf{q}}, a_{1,-\mathbf{q}}^{\dagger}, \ldots, a_{\mathcal{N}_{m},-\mathbf{q}}^{\dagger}\right)^{\mathrm{T}}$ and $\mathbf{V}^{(1)}$ is a $1 \times\left(2 \mathcal{N}_{m}\right)$ vector with elements $V_{\mu}^{(1)}=\sum_{\mu^{\prime}} V_{\mu \mu^{\prime}}^{(1)}$. 
Note that $\mathbf{x}_{\mathbf{q}}$ appears also explicitly in the quadratic part of the effective spin Hamiltonian, in the form

$$
\mathcal{H}_{\mathrm{LSW}}=\frac{S}{2} \sum_{\mathbf{q}} \mathbf{x}_{\mathbf{q}}^{\dagger} \cdot \mathbf{H}_{\mathbf{q}} \cdot \mathbf{x}_{\mathbf{q}},
$$

where $\mathbf{H}_{\mathbf{q}}$ is a $\left(2 \mathcal{N}_{m}\right) \times\left(2 \mathcal{N}_{m}\right)$ coupling matrix. This Hamiltonian is diagonalized via a standard Bogoliubov transformation, $\mathbf{x}_{\mathbf{q}}=\mathbf{T}_{\mathbf{q}} \cdot \mathbf{y}_{\mathbf{q}}$, where $\mathbf{T}_{\mathbf{q}}$ is the canonical transformation matrix, $\mathbf{y}_{\mathbf{q}}=\left(b_{1, \mathbf{q}}, \ldots, b_{\mathcal{N}_{m}, \mathbf{q}}, b_{1,-\mathbf{q}}^{\dagger}, \ldots, b_{\mathcal{N}_{m},-\mathbf{q}}^{\dagger}\right)^{\mathrm{T}}$, which leads to

$$
\mathcal{H}_{\mathrm{LSW}}=\sum_{\mathbf{q}} \sum_{\mu=1}^{\mathcal{N}_{m}} \omega_{\mu, \mathbf{q}}\left(b_{\mu, \mathbf{q}}^{\dagger} b_{\mu, \mathbf{q}}+\frac{1}{2}\right),
$$

where the new bosons $b_{\mu, \mathbf{q}}^{\dagger}$ describe the magnon excitations with frequencies $\omega_{\mu, \mathbf{q}}$.

Coming back to the Raman operator and expressing $\mathbf{x}_{\mathbf{q}=0}=\mathbf{T}_{\mathbf{q}=\mathbf{0}} \cdot \mathbf{y}_{\mathbf{q}=\mathbf{0}}$ in Eq. (42) leads to the following expression for the relevant, $\mathbf{q}=0$ part of the one-magnon Raman operator:

$$
\mathcal{R}_{1, \mathbf{q}=0}=\mathbf{M}^{(1)}(\mathbf{q}=0) \cdot \mathbf{y}_{\mathbf{q}=\mathbf{0}},
$$

where $\mathbf{M}^{(1)}(\mathbf{q}=0)=\mathbf{V}^{(1)} \cdot \mathbf{T}_{\mathbf{q}=\mathbf{0}}$. At zero temperature, it suffices to keep the terms involving $b_{\mu, \mathbf{q}=0}^{\dagger}$, since only the processes with a magnon creation on the $\mathcal{N}_{m}$ modes $(\mu, \mathbf{q}=0)$ are allowed. Therefore, the zero-temperature one-magnon Raman intensity [Eq. (38) with $\mathcal{R} \rightarrow \mathcal{R}_{1, \mathbf{q}=0}$ ] can be written as

$$
\mathcal{I}_{1}(\Omega) \propto \sum_{\mu}\left|\mathbf{M}_{\mathcal{N}_{m}+\mu}^{(1)}(\mathbf{q}=0)\right|^{2} \delta\left(\Omega-\omega_{\mu, \mathbf{q}=0}\right) .
$$

The above equation is the basis for the numerical calculation of the one-magnon Raman scattering intensity. The $\delta$ functions are treated by allowing for a small, but otherwise arbitrary Lorentzian broadening $\delta(x) \rightarrow \frac{1}{\pi} \frac{\eta}{x^{2}+\eta^{2}}$ with a small enough value for $\eta$.

\section{B. Two-magnon scattering}

The two-magnon scattering involves the second-order term in Eq. (37), which reduces to

$$
\begin{aligned}
\mathcal{R}_{2}= & \sum_{\mathbf{R},\left(\mu \mu^{\prime}\right)_{v}}\left\{V_{\mu+\mathcal{N}_{m}, \mu^{\prime}}^{(2)} a_{\mathbf{R}, \mu} a_{\mathbf{R}+\mathbf{t}_{\mu \mu^{\prime}}, \mu^{\prime}}\right. \\
& +V_{\mu+\mathcal{N}_{m}, v+\mathcal{N}_{m}}^{(2)} a_{\mathbf{R}, \mu} a_{\mathbf{R}+\mathbf{t}_{\mu \mu^{\prime}, \mu^{\prime}}^{\dagger}}^{\dagger}+V_{\mu, \mu}^{(2)} a_{\mathbf{R}, \mu}^{\dagger} a_{\mathbf{R}, \mu} \\
& \left.+V_{\mu^{\prime}, \mu^{\prime}}^{(2)} a_{\mathbf{R}+\mathbf{t}_{\mu \mu^{\prime}}, \mu^{\prime}}^{\dagger} a_{\mathbf{R}+\mathbf{t}_{\mu \mu^{\prime}}, \mu^{\prime}}+\text { H.c. }\right\},
\end{aligned}
$$

where the various prefactors $V^{(2)}$ can be obtained from Eq. (32), similarly to the one-magnon case (we do not, however, write them down here since the respective expressions are rather cumbersome). Using again the Fourier transform and symmetrizing with respect to $\mathbf{q} \rightarrow-\mathbf{q}$, we obtain

$$
\mathcal{R}_{2}=\sum_{\mathbf{q}} \mathbf{x}_{\mathbf{q}}^{\dagger} \cdot \mathbf{V}^{(2)}(\mathbf{q}) \cdot \mathbf{x}_{\mathbf{q}}=\sum_{\mathbf{q}} \mathbf{y}_{\mathbf{q}}^{\dagger} \cdot \mathbf{M}^{(2)}(\mathbf{q}) \cdot \mathbf{y}_{\mathbf{q}},
$$

where $\mathbf{V}^{(2)}(\mathbf{q})$ and $\mathbf{M}^{(2)}(\mathbf{q})=\mathbf{T}_{\mathbf{q}}^{\dagger} \cdot \mathbf{V}^{(2)} \cdot \mathbf{T}_{\mathbf{q}}$ are $\left(2 \mathcal{N}_{m}\right) \times$ $\left(2 \mathcal{N}_{m}\right)$ matrices.

While the operator $\mathcal{R}_{2}$ contains all combinations of the bilinear terms $b_{\mu, \mathbf{q}} b_{\mu^{\prime}, \mathbf{q}}^{\dagger}, b_{\mu, \mathbf{q}} b_{\mu^{\prime},-\mathbf{q}}, b_{\mu,-\mathbf{q}}^{\dagger} b_{\mu^{\prime}, \mathbf{q}}^{\dagger}, b_{\mu,-\mathbf{q}}^{\dagger} b_{\mu^{\prime},-\mathbf{q}}$, at

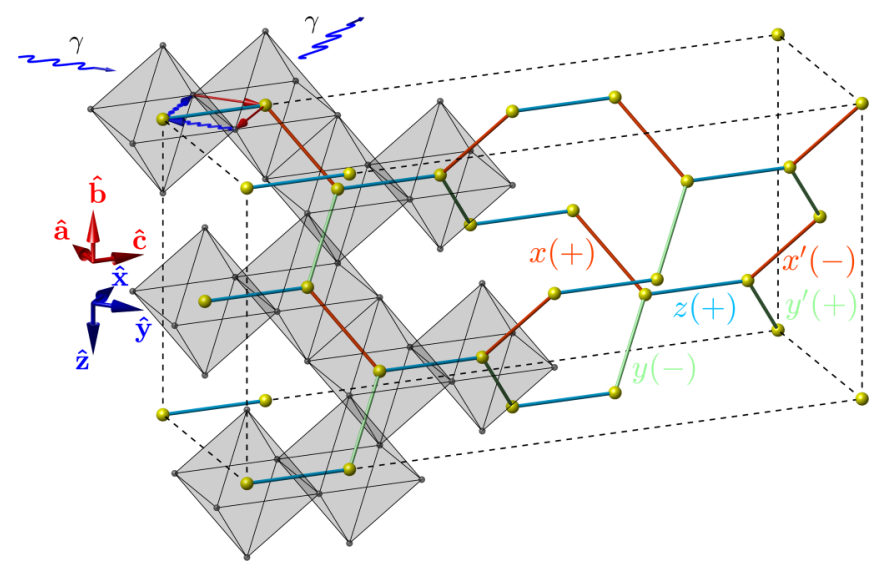

FIG. 5. Sketch of a hyperhoneycomb lattice of $\beta-\mathrm{Li}_{2} \mathrm{IrO}_{3}$. The orthorhombic unit cell is defined by the crystallographic axes $\{\hat{\mathbf{a}}, \hat{\mathbf{b}}, \hat{\mathbf{c}}\}$ related to the Cartesian axes $\{\hat{\mathbf{x}}, \hat{\mathbf{y}}, \hat{\mathbf{z}}\}$ appearing in the spin Hamiltonian by the following relations: $\hat{\mathbf{x}}=(\hat{\mathbf{a}}+\hat{\mathbf{c}}) / \sqrt{2}, \hat{\mathbf{y}}=(\hat{\mathbf{c}}-$ â $) / \sqrt{2}, \hat{\mathbf{z}}=-\hat{\mathbf{b}}$. The five NN bonds of the $J-K-\Gamma$ model are marked in red for $\boldsymbol{d} \in\left\{x, x^{\prime}\right\}$, green for $\boldsymbol{d} \in\left\{y, y^{\prime}\right\}$, and blue for $\boldsymbol{d} \in\{z\}$. Each octahedral denotes to the $\mathrm{IrO}_{6}$ cage.

zero temperature only those corresponding to two creation operators contribute to two-magnon scattering. Also, momentum conservation requires that the momenta of the two magnons must be opposite to each other, i.e., $\mathbf{q}^{\prime}=-\mathbf{q}$. This leads to

$$
\mathcal{R}_{2}=\sum_{\mathbf{q}} \sum_{\mu, \mu^{\prime}=1}^{\mathcal{N}_{m}} M_{\mu, \mu^{\prime}+\mathcal{N}_{m}}^{(2)}(\mathbf{q}) b_{\mu, \mathbf{q}}^{\dagger} b_{\mu^{\prime},-\mathbf{q}}^{\dagger}+\text { H.c. }
$$

Replacing in Eq. (38) (with $\mathcal{R} \rightarrow \mathcal{R}_{2}$ ) and using a Lehmann spectral representation into the relevant two-magnon space leads to the (zero temperature) two-magnon Raman intensity

$$
\mathcal{I}_{2}(\Omega) \propto \sum_{\mathbf{q}, \mu \mu^{\prime}}\left|M_{\mu, \mu^{\prime}+\mathcal{N}_{m}}^{(2)}(\mathbf{q})\right|^{2} \delta\left(\Omega-\omega_{\mu}(\mathbf{q})-\omega_{\mu^{\prime}}(\mathbf{q})\right)
$$

which is the basis for our numerical calculations (with the appropriate Lorentzian broadening as in the one-magnon case).

\section{APPLICATION TO $\beta$ - $\mathrm{Li}_{2} \mathrm{IrO}_{3}$}

We are now ready to apply the theory developed in the previous sections to compute the Raman intensity for $\beta-\mathrm{Li}_{2} \mathrm{IrO}_{3}$. This compound crystallizes in a hyperhoneycomb structure, with a conventional orthorhombic unit cell defined by the crystallographic axes $\{\hat{\mathbf{a}}, \hat{\mathbf{b}}, \hat{\mathbf{c}}\}$, see Fig. 5 .

At zero field, $\beta$ - $\mathrm{Li}_{2} \mathrm{IrO}_{3}$ orders magnetically below $T_{N}=$ $38 \mathrm{~K}$. The magnetic structure is characterized by a noncoplanar, incommensurate (IC) modulation, with propagation wave vector $\mathbf{Q}=(0.57,0,0)$ (in orthorhombic frame units), and two counter-rotating sets of moments [48]. According to previous theoretical works, the magnetism of $\beta-\mathrm{Li}_{2} \mathrm{IrO}_{3}$ can be accurately described by the $J-K-\Gamma$ model of Eq. (5) with $J=0.4 \mathrm{meV}, K=-18 \mathrm{meV}$ and $\Gamma=-10 \mathrm{meV}[56,57,65-69]$. Furthermore, it has been shown $[65,66]$ that the IC order of $\beta$ - $\mathrm{Li}_{2} \mathrm{IrO}_{3}$ can be treated as a long-distance twisting of a nearby commensurate period-three state with $\mathbf{Q}=\frac{2}{3} \hat{\mathbf{a}}$ (in units $\frac{2 \pi}{a}$ ). This state is amenable to a semianalytical treatment, which delivers a very accurate representation of the ground 


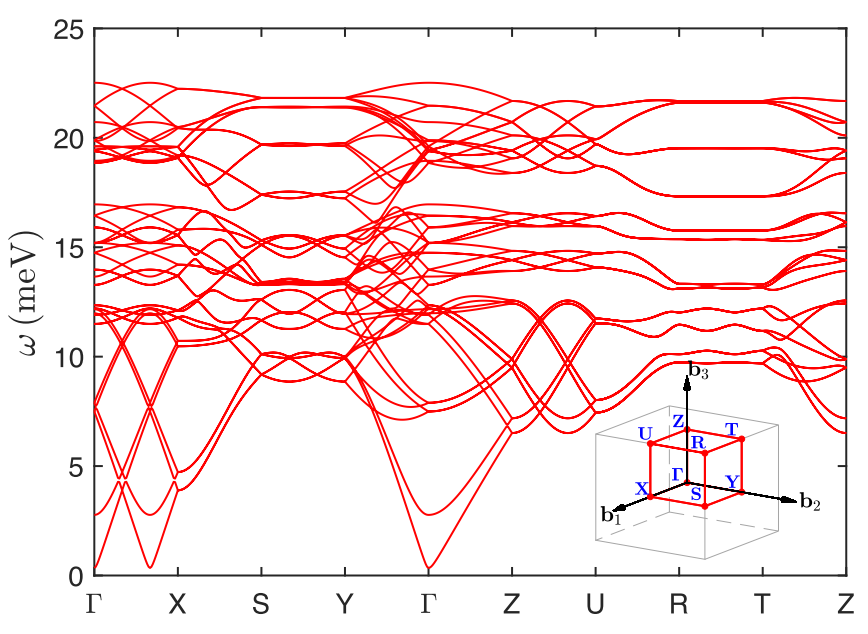

FIG. 6. Linear spin wave spectrum along a high symmetry path in the Brillouin zone of the orthorhombic unit cell (inset).

state properties and the magnon excitation spectrum [65-68]. For the latter, we take a magnetic supercell composed of three orthorhombic unit cells along the a axis, and thus $\mathcal{N}_{m}=48$ spin sites [65]. The ensuing 48 magnon branches delivered by the numerical diagonalization of $\mathcal{H}_{\mathrm{LSW}}$ is shown in Fig. 6 along a high symmetry path in the Brillouin zone of the orthorhombic unit cell $[65,67]$. Note that the spectrum features a nonzero spin gap, which reflects the presence of anisotropic exchange interactions and the absence of continuous translational symmetry.

Turning to the Raman operator and Eq. (32), we will need the numerical values of the various quantities appearing in $\mathrm{Eq}$. (33). These include the vectors $\mathbf{d}_{i j}$ and $\mathbf{d}_{i j}^{\perp}$ of Eq. (20), as well as the parameters $J^{(2)}, J^{\prime(4)}, K^{(2)}, K^{(4)}, K^{\prime(4)}, \Gamma^{(3)}$, and $h_{\Gamma}^{(3)}$. For the former, there are five types of bonds in $\beta-\mathrm{Li}_{2} \mathrm{IrO}_{3}$, labeled by $x, x^{\prime}, y, y^{\prime}$, and $z$ (see Fig. 5), with

$$
\begin{aligned}
\mathbf{d}_{x} & =\mathbf{d}_{x^{\prime}}^{\perp}=\frac{1}{2}[1, \sqrt{2},-1], \\
\mathbf{d}_{x}^{\perp} & =\mathbf{d}_{x^{\prime}}=\frac{1}{2}[1,-\sqrt{2},-1], \\
\mathbf{d}_{y} & =\mathbf{d}_{y^{\prime}}^{\perp}=-\frac{1}{2}[1, \sqrt{2}, 1], \\
\mathbf{d}_{y}^{\perp} & =\mathbf{d}_{y^{\prime}}=\frac{1}{2}[-1, \sqrt{2},-1], \\
\mathbf{d}_{z} & =[0,0,1], \mathbf{d}_{z}^{\perp}=[1,0,0],
\end{aligned}
$$

in the orthorhombic frame. The remaining parameters appearing in Eq. (32) depend on the hopping matrix elements $t_{1}$, $t_{2}=t^{2} / \Delta_{\mathrm{pd}}$, and $t_{3}$, where $\Delta_{\mathrm{pd}}$ is the charge transfer energy, and the interaction terms $U_{2}, J_{H}$, and $\lambda$. Fixing the latter to the typical values of $U_{2}=1.8 \mathrm{eV}, J_{H}=0.4 \mathrm{eV}$, and $\lambda=0.4 \mathrm{eV}$, allows us to adjust $t_{1}, t_{2}$, and $t_{3}$ so that we reproduce the values of $J, K, \Gamma$ mentioned above. This gives $t_{1}=-0.042 \mathrm{eV}$, $t_{2}=0.332 \mathrm{eV}$, and $t_{3}=0.190 \mathrm{eV}$, which are within the typical range of density functional theory (DFT) calculations, see, e.g., Ref. [70]. Furthermore, we assume that the incoming light is off resonance and neglect the frequency of the incoming light, i.e., we set $\omega_{\text {in }}=0$ in the expressions for the Raman operator. With these assumptions and numerical estimates we arrive at: $J^{(2)}=0.4 \mathrm{meV}, J^{\prime(4)}=-101.797 \mathrm{meV}$, $K^{(2)}=3.49 \mathrm{meV}, K^{(4)}=-21.49 \mathrm{meV}, K^{\prime(4)}=210.757 \mathrm{meV}$,
$\Gamma^{(3)}=-10 \mathrm{meV}$, and $h_{\Gamma}^{(3)}=14.615 \mathrm{meV}$. Quite remarkably, the parameters $J^{\prime(4)}$ and $K^{\prime(4)}$ that are partly responsible for the non-Loudon-Fleury Raman scattering, have much larger magnitude compared to the corresponding values of $J^{(2)}$ and $K^{(2)}+K^{(4)}$ of the Loudon-Fleury terms. Similarly, the magnitude of $h_{\Gamma}^{(3)}$, which does not have any analog in the spin Hamiltonian, is appreciably high as well. This tells us that the Raman intensity (which scales quadratically with the parameters) is dominated by the non-Loudon-Fleury scattering terms. This significant result will be demonstrated explicitly below.

Having the numerical values of the various quantities appearing in Eq. (32) we can now calculate the one- and twomagnon Raman intensity of $\beta-\mathrm{Li}_{2} \mathrm{IrO}_{3}$ using Eqs. (46) and (50), respectively. In particular, we will focus on scattering geometries corresponding to incoming and outgoing light polarizations along the orthorhombic crystal axes. Among these are the diagonal polarization channels where $\boldsymbol{\varepsilon}_{\text {in }}=\boldsymbol{\varepsilon}_{\text {out }}=\mathbf{a}$ or $\mathbf{b}$ or $\mathbf{c}$ (which we will label by $\mathcal{R}^{a a}, \mathcal{R}^{b b}$, and $\mathcal{R}^{c c}$, respectively), as well as off-diagonal polarization channels where, e.g., $\boldsymbol{\varepsilon}_{\text {in }}=\mathbf{a}$ and $\boldsymbol{\varepsilon}_{\text {out }}=\mathbf{b}$ (which we label as $\mathcal{R}^{a b}$ ), etc. A symmetry analysis based on the $D_{2 h}$ point group ${ }^{2}$ shows that $\mathcal{R}^{a a}, \mathcal{R}^{b b}$, and $\mathcal{R}^{c c}$ transform according to the $A_{g}$ irreducible representation, while $\mathcal{R}^{a b}, \mathcal{R}^{a c}$, and $\mathcal{R}^{b c}$ transform as $B_{1 g}, B_{2 g}$, and $B_{3 g}$, respectively $[21,71]$.

One-magnon response. Figures 7(a)-7(b) show the onemagnon Raman scattering intensities in the polarization channels mentioned above, as obtained from numerical calculations based on Eq. (46) with a Lorentzian broadening parameter $\eta=0.5 \mathrm{meV}$. To disentangle the contributions coming from the non-Loudon-Fleury terms we perform calculations with [Fig. 7(b)] and without [Fig. 7(a)] these terms. A quick inspection of the intensity scales in the two panels demonstrates the dramatic impact of the non-Loudon-Fleury terms announced above, namely that these terms dominate the scattering. Another significant ramification of these terms is that the sharp peak at $\Omega \sim 3 \mathrm{meV}$, appearing in the $a c$ polarization channel in Fig. 7(b), is absent from Fig. 7(a). This one-magnon $\mathbf{q}=0$ peak, therefore, originates from the nonLoudon-Fleury terms. We have checked, in particular, that this peak stems from the magnetic dipole-active terms $\propto h_{\Gamma}^{(3)}$, see inset of Fig. 7(c). It is furthermore noteworthy that this peak is absent in the remaining polarization channels shown in Fig. 7(b), which can be used as a smoking-gun diagnostic feature in experiments. ${ }^{3}$

The strong polarization dependence is not special to the low-energy peak, but manifests in the higher-energy part of the response as well, as shown in Fig. 7(b). Comparing with Fig. 7(a), the non-Loudon-Fleury terms play a decisive role, as they modify significantly the relative intensity and overall shape of the high-energy peaks.

\footnotetext{
${ }^{2}$ The zero-field ground state of $\beta-\mathrm{Li}_{2} \mathrm{IrO}_{3}$ [48-50] breaks some of the symmetries of the lattice [65,67], but the point group of this state is isomorphic to $D_{2 h}$ so we can still use this group for the analysis of the Raman scattering channels.

${ }^{3}$ This peak has, in fact, been observed experimentally [37] and will be discussed elsewhere.
} 

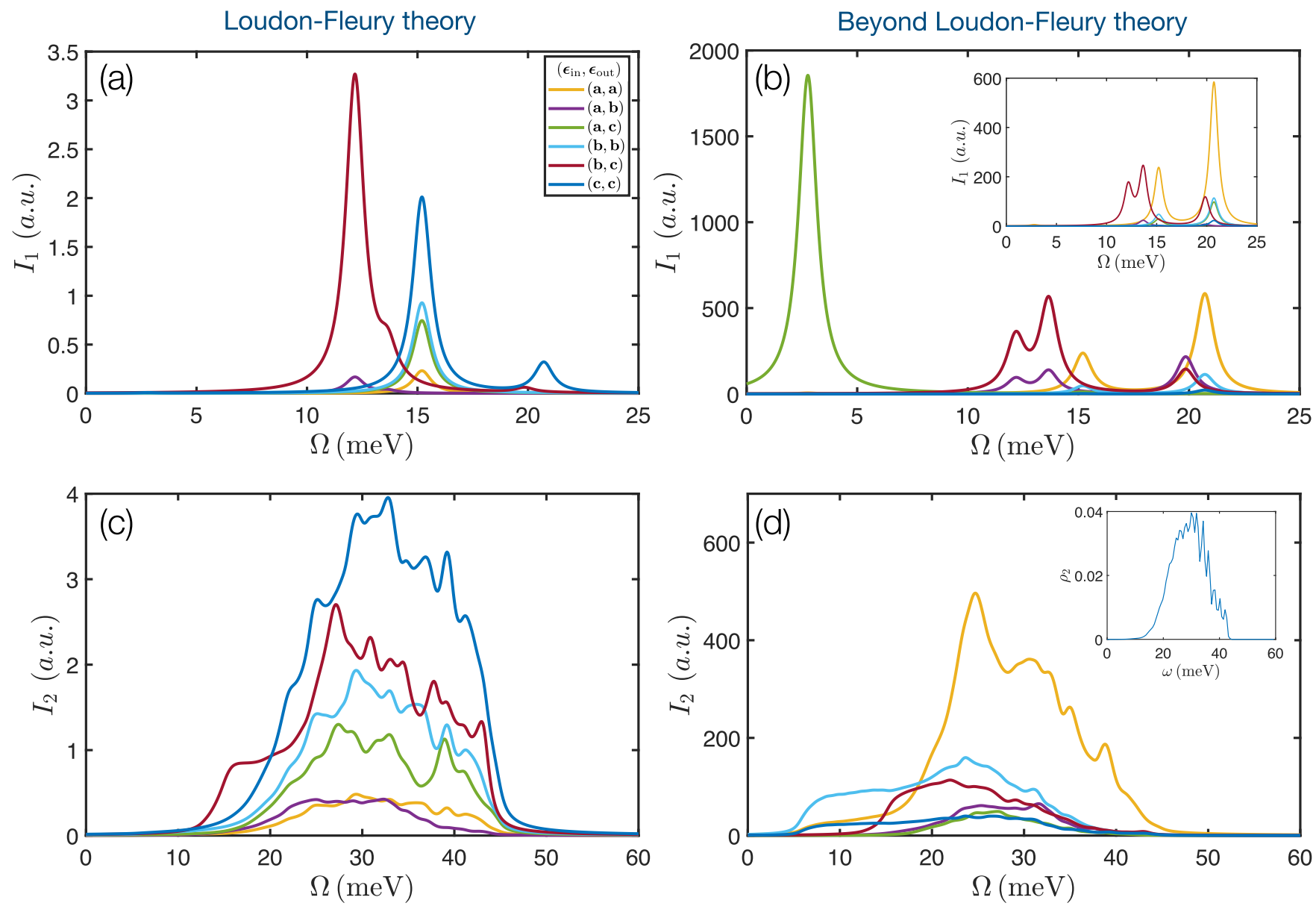

FIG. 7. One-magnon (a)-(b) and two-magnon (c)-(d) Raman intensities, computed with [(b) and (d)] and without [(a) and (c)] taking into account the non-Loudon-Fleury scattering terms, see main text. Lines with different colors correspond to different polarization channels (with the color scheme being consistent across all the panels). The inset of (b) shows the Raman response without the non-Loudon-Fleury, magnetic-dipole term $\propto h_{\Gamma}^{(3)}$. The inset of (d) shows the two-magnon density of states $\rho_{2}$ of Eq. (52).

Two-magnon response. We now turn to the two-magnon intensities shown in Figs. 7(c)-7(d). As above, we disentangle the contributions from the non-Loudon-Fleury terms by performing calculations with [Fig. 7(d)] and without [Fig. 7(c)] these terms. The intensities are computed using Eq. (50), where the sum over $\mathbf{q}$ in Eq. (50) is carried out on a finite-size grid of $25200 \mathbf{q}$ points within the magnetic Brillouin zone, and the Lorentzian broadening parameter is chosen to be $\eta=0.6 \mathrm{meV}$.

Quite generally, the two-magnon response features a broad continuum, mainly due to the fact that Eq. (50) involves a sum over all $\mathbf{q}$ modes. This sum has the form of a convolution between a polarization-dependent weight $\left|M^{(2)}(\mathbf{q})\right|^{2}$ and the two-magnon density of states, defined as

$$
\rho_{2}(\omega)=\sum_{\mu, \mu^{\prime}, \mathbf{q}} \delta\left(\omega-\omega_{\mu}(\mathbf{q})-\omega_{\mu^{\prime}}(-\mathbf{q})\right) .
$$

The latter is calculated using a histogram method and is shown for comparison in the inset of Fig. 7(d) and reproduces well the bandwidth and overall shape of the response.

A quick inspection of the intensity scales in Figs. 7(c) and 7(d) shows that the two-magnon intensity too is dominated by the non-Loudon-Fleury terms. Furthermore, these terms change significantly the relative intensities of the various polarization channels. For example, the $\mathcal{R}^{a a}$ channel features the largest response, unlike the computed intensities based on the Loudon-Fleury terms alone [Fig. 7(c)]. These significant changes come with distinctive features, which can again be tested experimentally.

Here we also note that the two-magnon intensities shown in Figs. 7(c)-7(d) are obtained without taking into account the effects of the final-state magnon-magnon interactions [36], which might be not small given the complex nature of the magnetic ordering in $\beta-\mathrm{Li}_{2} \mathrm{IrO}_{3}$. In principle, their effect can be taken into account by computing the vertex corrections to the bare Raman vertex in Eq. (49), although this is a technically rather tedious task due to the large number of magnon bands in $\beta-\mathrm{Li}_{2} \mathrm{IrO}_{3}$. Qualitatively, we expect that these corrections can lead to a shifting of the two-magnon peaks to lower energies and to the formation of an even broader continuum at the higher energies, similarly to the cases considered in Refs. [4] and [10].

Finally, we point out that the numerical results presented here correspond to the case where the incoming light is off resonance. The case of resonance, i.e., when the frequency of 
the incoming photon is comparable to the charge gap, requires further analysis $[4,5]$.

\section{DISCUSSION}

We have revisited the theory of magnetic Raman scattering in Mott insulators with strong spin-orbit coupling with a special focus on Kitaev materials. A detailed consideration of the precise photon-assisted, virtual hopping processes that contribute to the magnetic Raman scattering reveals that the Raman vertex $\mathcal{R}$ contains terms beyond those appearing in the traditional Loudon-Fleury theory. Quite remarkably, these non-Loudon-Fleury terms are shown to dominate the scattering intensity in the three-dimensional Kitaev material $\beta-\mathrm{Li}_{2} \mathrm{IrO}_{3}$ by at least two orders of magnitude. In addition, the non-Loudon-Fleury terms give rise to a qualitative modification of the polarization dependence, with distinctive signatures that can be tested experimentally. Most saliently, in $\beta$ - $\mathrm{Li}_{2} \mathrm{IrO}_{3}$ the non-Loudon-Fleury terms give rise to a sharp magnetic dipole-active magnon peak at low energies, which is absent in the traditional Loudon-Fleury theory. This peak has been observed recently in the predicted $a c$-polarization channel [37], lending strong support to the importance of the non-Loudon-Fleury terms. The peak is shown to arise from virtual tunneling processes involving both direct and ligand mediated paths. These processes are of similar type with the ones leading to the symmetric off-diagonal interaction $\Gamma$, but, in the Raman vertex, they take the form of a bond-directional magnetic dipole term. In particular, these processes involve an intermediate hopping to the ligand (oxygen in $\beta-\mathrm{Li}_{2} \mathrm{IrO}_{3}$ ), which does not conserve the projection of the total pseudospin along the corresponding axis (e.g., $S_{i}^{z}+S_{j}^{z}$ for the $z$ type of bonds).

On a broader perspective, we would also like to emphasise that our theory is fully applicable to any strong spin-orbit coupled Mott insulator, in which the magnetic moments $j_{\text {eff }}=$ $1 / 2$ come from the five electrons (or one hole) on the $t_{2 g}$ orbitals. For Kitaev materials, in particular, we even expect similar quantitative results with the ones presented here for $\beta$ - $\mathrm{Li}_{2} \mathrm{IrO}_{3}$, as the underlying local geometry (and the effective spin Hamiltonian description) of $\beta-\mathrm{Li}_{2} \mathrm{IrO}_{3}$ is common in all Kitaev materials. Specifically, we expect the same type of non-Loudon-Fleury terms (including the magnetic dipole term $\propto h_{\Gamma}^{(3)}$ ) to be present generically across all Kitaev materials, and we also anticipate that these will dominate the scattering intensity, given the similar order of magnitude of the microscopic parameters $U_{2}, J_{H}, \lambda$, and $\Delta_{\mathrm{pd}}$. The presented analysis therefore underpins a drastic change of paradigm for the understanding of Raman scattering in materials with strong spin-orbit coupling and multiple exchange paths. In addition, it calls for a general reevaluation of Raman scattering in Kitaev materials of current interest, as this would help to elucidate their correct microscopic description and their relative proximity to the sought-after quantum spin liquid.

Note added. We recently became aware that a modification of exchange interactions similar to the one presented here for $\mathcal{R}$ has been discussed for Kitaev materials under magnetic field [72] and circularly polarized light [73]. In particular, Ref. [72] reports an effective magnetic field term similar to $h_{\Gamma}^{(3)}$, which also arises from mixed hopping terms.

\section{ACKNOWLEDGMENTS}

We thank Kenneth Burch and Yiping Wang for helpful discussions and for sharing with us unpublished Raman data on $\beta-\mathrm{Li}_{2} \mathrm{IrO}_{3}$. The work by Y.Y., M.L., and N.B.P. was supported by the U.S. Department of Energy, Office of Science, Basic Energy Sciences under Award No. DE-SC0018056. We also acknowledge the support of the Minnesota Supercomputing Institute (MSI) at the University of Minnesota. N.B.P. and Y.Y. are thankful for the hospitality of Kavli Institute for Theoretical Physics and the support of the National Science Foundation under Grant No. NSF PHY-1748958.

\section{APPENDIX: TECHNICAL DETAILS OF THE DERIVATION OF THE RAMAN OPERATOR}

\section{Raman operator from the processes involving the direct hoppings}

Since it is more convenient to represent the hopping matrix $\mathcal{H}_{t}$ in the orbital basis, we project the $j_{\text {eff }}=1 / 2$ pseudospin degree of freedom to the orbital basis using

$$
\begin{aligned}
& \left|j_{\mathrm{eff}}^{z}=+\frac{1}{2}\right\rangle=-\frac{1}{\sqrt{3}}(|X, \downarrow\rangle+i|Y, \downarrow\rangle+|Z, \uparrow\rangle) \\
& \left|j_{\mathrm{eff}}^{z}=-\frac{1}{2}\right\rangle=-\frac{1}{\sqrt{3}}(|X, \uparrow\rangle-i|Y, \uparrow\rangle-|Z, \downarrow\rangle) .
\end{aligned}
$$

Next we notice that there are 15 intermediate states $\left|D_{\mu}\right\rangle$ with two holes on the iridium ion, which can be obtained by the diagonalization of $\mathcal{H}_{\text {int }}$ and can be easily written in the two-hole orbital basis at the zero SOC limit $\lambda \rightarrow 0$. Explicitly, we denote the two-hole orbital basis in the way given in the Table IV.

There are several processes that contribute to the spin interaction and we consider those involving the direct hopping only. These processes give rise to the effective spin coupling at the second order of perturbation theory. The half of the direct hopping path projected into all possible orbital channels is shown in Fig. 8. In this process, the hopping starts from the $\operatorname{Ir}_{1}$ ion from one of the $j_{\text {eff }}^{z}= \pm 1 / 2$ states and ends on $\operatorname{Ir}_{2}$ ion in one of the 15 states belonging to the two-hole orbital basis.

For example, in Fig. 8(a), we show the action of the hopping term on the $\left|\psi_{1}\right\rangle$ state, which we denote as $\mathcal{H}_{t}\left|\psi_{1}\right\rangle \equiv \mathcal{H}_{t}|+1 / 2 ;+1 / 2\rangle$. The hole on $\operatorname{Ir}_{1}$ is first projected to the orbital basis $|X \downarrow\rangle,|Y \downarrow\rangle$ and $|Z \uparrow\rangle$. Each of these states overlaps with the two-hole states on $\operatorname{Ir}_{2}$. Recalling that we are interested only on those two-hole states, in which one hole is the original hope on the $\left|j_{\text {eff }}^{z}=1 / 2\right\rangle_{2}$, we should take into account the projection of $\left|j_{\text {eff }}^{z}=1 / 2\right\rangle_{2}$ to the orbital basis. The product of weights from the projection gives the weights for each channel of hopping in the orbital basis. The sum of all the contributions allows us to explicitly compute the matrix

TABLE IV. Intermediate, two-hole (Slater determinant) states.

\begin{tabular}{lll}
\hline \hline$|X \uparrow, X \downarrow\rangle \equiv|1\rangle$ & $|Y \uparrow, Z \uparrow\rangle \equiv|6\rangle$ & $|X \downarrow, Y \uparrow\rangle \equiv|11\rangle$ \\
$|Y \uparrow, Y \downarrow\rangle \equiv|2\rangle$ & $|X \downarrow, Y \downarrow\rangle \equiv|7\rangle$ & $|X \uparrow, Z \downarrow\rangle \equiv|12\rangle$ \\
$|Z \uparrow, Z \downarrow\rangle \equiv|3\rangle$ & $|X \downarrow, Z \downarrow\rangle \equiv|8\rangle$ & $|X \downarrow, Z \uparrow\rangle \equiv|13\rangle$ \\
$|X \uparrow, Y \uparrow\rangle \equiv|4\rangle$ & $|Y \downarrow, Z \downarrow\rangle \equiv|9\rangle$ & $|Y \uparrow, Z \downarrow\rangle \equiv|14\rangle$ \\
$|X \uparrow, Z \uparrow\rangle \equiv|5\rangle$ & $|X \uparrow, Y \downarrow\rangle \equiv|10\rangle$ & $|Y \downarrow, Z \uparrow\rangle \equiv|15\rangle$ \\
\hline \hline
\end{tabular}


(a)

(b)

$$
\begin{aligned}
& \left|+\frac{1}{2},+\frac{1}{2}\right\rangle \stackrel{\text { direct hopping }}{\longrightarrow}\left\{\begin{array} { c } 
{ \frac { t _ { 1 } - t _ { 3 } } { 3 } | 1 3 \rangle } \\
{ \frac { i ( t _ { 1 } - t _ { 3 } ) } { 3 } | 1 5 \rangle }
\end{array} | - \frac { 1 } { 2 } , - \frac { 1 } { 2 } \rangle \stackrel { \text { direct hopping } } { \longrightarrow } \left\{\begin{array}{c}
\frac{t_{3}-t_{1}}{3}|12\rangle \\
\frac{i\left(t_{1}-t_{3}\right)}{3}|14\rangle
\end{array}\right.\right. \\
& \left.+\frac{1}{2},-\frac{1}{2}\right\rangle \stackrel{\text { direct hopping }}{\longrightarrow}\left\{\begin{array}{c}
-\frac{t_{1}}{3}|1\rangle \\
-\frac{t_{1}}{3}|2\rangle \\
-\frac{t_{3}}{3}|3\rangle \\
-\frac{t_{3}}{3}|5\rangle \\
\frac{i}{3} t_{3}|6\rangle \\
-\frac{t_{1}}{3}|8\rangle \\
-\frac{i}{3} t_{1}|9\rangle \\
-\frac{i}{3} t_{1}|10\rangle \\
-\frac{i}{3} t_{1}|11\rangle
\end{array}\right.
\end{aligned}
$$

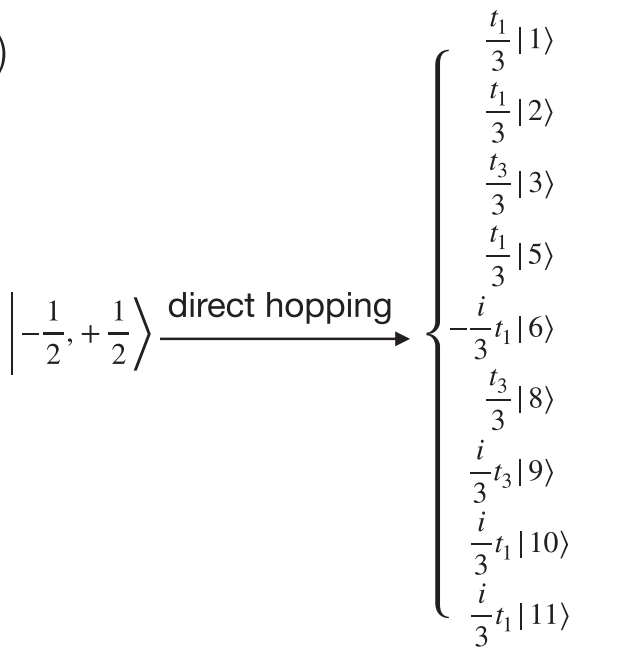

FIG. 8. Direct hopping from one-hole state on $\operatorname{Ir}_{1}$ and $\operatorname{Ir}_{2}$ to the intermediate two-hole states (denoted in Table IV) on $\mathrm{Ir}_{2}$ from one of four possible $\left|\psi_{n}\right\rangle$ ground states: (a) $\left|\psi_{1}\right\rangle \equiv|+1 / 2 ;+1 / 2\rangle$, (b) $\left|\psi_{4}\right\rangle \equiv|-1 / 2 ;-1 / 2\rangle$, (c) $\left|\psi_{3}\right\rangle \equiv|+1 / 2 ;-1 / 2\rangle$, and (d) $\left|\psi_{2}\right\rangle \equiv|-1 / 2 ;+1 / 2\rangle$.

element $\left\langle D_{\mu}\left|\mathcal{H}_{t}\right| \psi_{1}\right\rangle$, where $\left|D_{\mu}\right\rangle$ denotes the two-hole intermediate state in the $\mu=1,2, \ldots, 15$ th state entering into Eq. (21). The same calculation can be performed for other states $\mathcal{H}_{t}|n\rangle$ as illustrated in Figs. 8(b)-8(d). Next we compute $\left\langle D_{\mu}\left|\mathcal{H}_{t}\right| n\right\rangle$ and $\left\langle D_{\mu}\left|\mathcal{H}_{t}\right| m\right\rangle^{*}$ to form the complete hopping path. The process starting from $\mathrm{Ir}_{2}$ gives exactly the same result.
When we consider the Raman operator, we should recall that the incoming light must couple to the hopping on the first bond, and the outgoing light must couple to the hopping on the last bond of the path. With only two hopping bonds in the direct hopping path, the polarization factor can only be $\left(\boldsymbol{\varepsilon}_{\text {in }} \cdot \mathbf{d}_{i j}\right)\left[\boldsymbol{\varepsilon}_{\text {out }} \cdot\left(-\mathbf{d}_{i j}\right)\right]$, and so we can compute the Raman operator originated from the direct hopping as

$$
\mathcal{R}_{\langle i j\rangle_{z}}^{\mathrm{dir}}=-2 \zeta\left(\boldsymbol{\varepsilon}_{\mathrm{in}} \cdot \mathbf{d}_{i j}\right)\left(\boldsymbol{\varepsilon}_{\mathrm{out}} \cdot \mathbf{d}_{i j}\right) \sum_{n, n^{\prime}} \sum_{\mu} \frac{\left\langle D_{\mu}\left|\mathcal{H}_{t, i j}\right| \psi_{n}\right\rangle^{*} \cdot\left\langle D_{\mu}\left|\mathcal{H}_{t, i j}\right| \psi_{n^{\prime}}\right\rangle}{2 E_{1 \mathrm{~h}}+\omega_{\mathrm{in}}-\left(E_{2 \mathrm{~h}}+E_{0 \mathrm{~h}}\right)}\left|\psi_{n}\right\rangle\left\langle\psi_{n^{\prime}}\right|,
$$

where $\boldsymbol{\varepsilon}_{\text {out }}$ as the outgoing light polarization, and $\left|\psi_{n}\right\rangle,\left|\psi_{n^{\prime}}\right\rangle$ again represent the four states of the low-energy sector of two magnetic ions, namely, $\left|\frac{1}{2}, \frac{1}{2}\right\rangle,\left|\frac{1}{2},-\frac{1}{2}\right\rangle,\left|-\frac{1}{2}, \frac{1}{2}\right\rangle,\left|-\frac{1}{2},-\frac{1}{2}\right\rangle$.

\section{Raman operator from the processes involving the oxygen-mediated hopping}

There are eight paths that include the oxygen-mediated hopping. These processes give rise to the effective spin coupling at the fourth order of perturbation theory, and the sum over all eight contributions gives us the superexchange Hamiltonian with the dominant Kitaev interaction $[55,61,63]$. Each of these paths also gives the contribution to the Raman operator $(24)$, which apart from the corresponding polarization prefactor is proportional to

$$
\begin{aligned}
\mathcal{H}_{\ell}^{O} & =\mathcal{H}_{t} \mathcal{G}_{O} \mathcal{H}_{t} \mathcal{G}_{D} \mathcal{H}_{t} \mathcal{G}_{O} \mathcal{H}_{t}=\mathcal{H}_{t} \frac{\left|O_{v^{\prime}}\right\rangle\left\langle O_{\nu^{\prime}}\right|}{\omega_{\mathrm{in}}-\Delta_{\mathrm{pd}}} \mathcal{H}_{t}\left(\sum_{\mu} \frac{\left|D_{\mu}\right\rangle\left\langle D_{\mu}\right|}{2 E_{1 \mathrm{~h}}+\omega_{\mathrm{in}}-\left(E_{2 \mathrm{~h}}+E_{0 \mathrm{~h}}\right)}\right) \mathcal{H}_{t} \frac{\left|O_{v}\right\rangle\left\langle O_{v}\right|}{\omega_{\mathrm{in}}-\Delta_{\mathrm{pd}}} \mathcal{H}_{t} \\
& =\sum_{n, n^{\prime}} \sum_{\mu} \frac{\left(\left\langle\psi_{n}\left|\mathcal{H}_{t}\right| O_{v^{\prime}}\right\rangle\left\langle O_{v^{\prime}}\left|\mathcal{H}_{t}\right| D_{\mu}\right\rangle\right)\left(\left\langle D_{\mu}\left|\mathcal{H}_{t}\right| O_{\nu}\right\rangle\left\langle O_{v}\left|\mathcal{H}_{t}\right| \psi_{n^{\prime}}\right\rangle\right)}{\left[2 E_{\mathrm{lh}}+\omega_{\mathrm{in}}-\left(E_{2 \mathrm{~h}}+E_{0 \mathrm{~h}}\right)\right]\left(\omega_{\mathrm{in}}-\Delta_{\mathrm{pd}}\right)^{2}}\left|\psi_{n}\right\rangle\left\langle\psi_{n^{\prime}}\right| .
\end{aligned}
$$

The choice of $\left|O_{v}\right\rangle$ determines whether the upper path or the lower path is considered [see Figs. 9(a) and 9(f), respectively]. The explicit construction of $\mathcal{H}_{t}\left|O_{v}\right\rangle\left\langle O_{v}\left|\mathcal{H}_{t}\right| \psi_{n^{\prime}}\right\rangle$ with all projection factors is obtained on the similar way as for the paths with the direct hopping only and is shown in Fig. 9. 
(a)

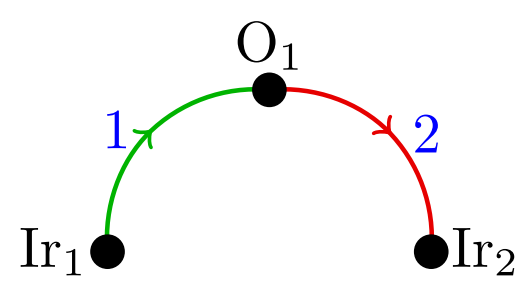

(b)

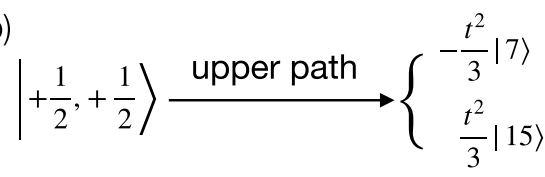

(d)

$\left|+\frac{1}{2},-\frac{1}{2}\right\rangle \stackrel{\text { upper path }}{\longrightarrow}\left\{\begin{array}{c}-\frac{t^{2}}{3}|10\rangle \\ \frac{i}{3} t^{2}|2\rangle \\ -\frac{t^{2}}{3}|9\rangle\end{array}\right.$

(g)

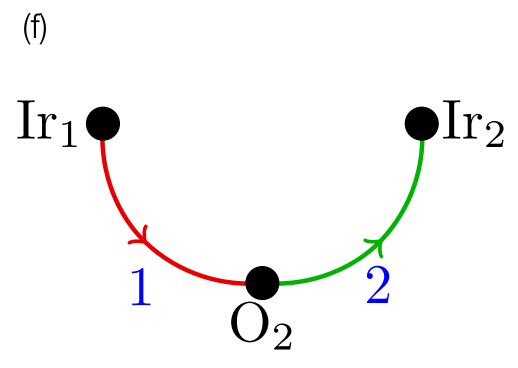

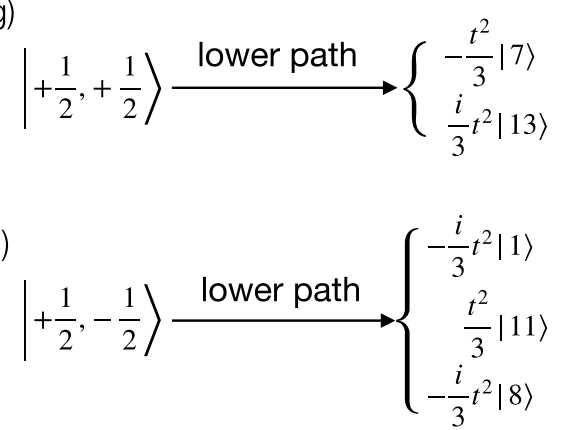

(c)

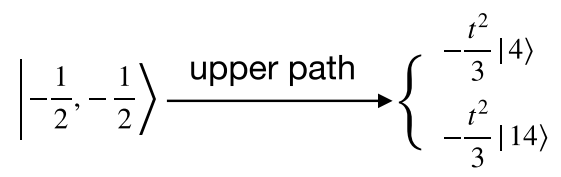

(e)

$\left|-\frac{1}{2},+\frac{1}{2}\right\rangle \stackrel{\text { upper path }}{\longrightarrow}\left\{\begin{array}{c}-\frac{t^{2}}{3}|11\rangle \\ \frac{i}{3} t^{2}|2\rangle \\ \frac{t^{2}}{3}|6\rangle\end{array}\right.$

(h)

$$
\left|-\frac{1}{2},-\frac{1}{2}\right\rangle \stackrel{\text { lower path }}{\longrightarrow}\left\{\begin{array}{c}
-\frac{t^{2}}{3}|4\rangle \\
\frac{i}{3} t^{2}|12\rangle
\end{array}\right.
$$

(j)

$$
\left|-\frac{1}{2},+\frac{1}{2}\right\rangle \stackrel{\text { lower path }}{\longrightarrow}\left\{\begin{array}{c}
-\frac{i}{3} t^{2}|1\rangle \\
\frac{t^{2}}{3}|10\rangle \\
\frac{i}{3} t^{2}|12\rangle
\end{array}\right.
$$

FIG. 9. Oxygen mediated hopping from one-hole state on $\operatorname{Ir}_{1}$ and $\operatorname{Ir}_{2}$ to the intermediate two-hole states (denoted in Table IV) on $\operatorname{Ir}_{2}$ from one of four possible $\left|\psi_{n}\right\rangle$ ground states. (b)-(e) represent the hopping via the upper path (a) and (g)-(j) represent the hopping via the lower path (f).

Once we have $\left\langle D_{\mu}\left|\mathcal{H}_{t}\right| O_{v}\right\rangle\left\langle O_{v}\left|\mathcal{H}_{t}\right| \psi_{n^{\prime}}\right\rangle$ computed for all $v$ and $\beta$, we can assemble the hopping path for the oxygen-mediated hopping as

$$
\left(\left\langle D_{\mu}\left|\mathcal{H}_{t}\right| O_{v^{\prime}}\right\rangle\left\langle O_{v^{\prime}}\left|\mathcal{H}_{t}\right| \psi_{n}\right\rangle\right)^{*} \cdot\left(\left\langle D_{\mu}\left|\mathcal{H}_{t}\right| O_{v}\right\rangle\left\langle O_{v}\left|\mathcal{H}_{t}\right| \psi_{n^{\prime}}\right\rangle\right) .
$$

This gives us the following expressions for $\mathcal{H}_{\ell}^{O}$ :

$$
\mathcal{H}_{\ell}^{O}=\sum_{n, n^{\prime}} \sum_{\mu} \frac{\left(\left\langle D_{\mu}\left|\mathcal{H}_{t}\right| O_{v^{\prime}}\right\rangle\left\langle O_{v^{\prime}}\left|\mathcal{H}_{t}\right| \psi_{n}\right\rangle\right)^{*} \cdot\left(\left\langle D_{\mu}\left|\mathcal{H}_{t}\right| O_{v}\right\rangle\left\langle O_{v}\left|\mathcal{H}_{t}\right| \psi_{n^{\prime}}\right\rangle\right)}{\left(2 E_{1 \mathrm{~h}}+\omega_{\mathrm{in}}-\left(E_{2 \mathrm{~h}}+E_{0 \mathrm{~h}}\right)\right)\left(\omega_{\mathrm{in}}-\Delta_{\mathrm{pd}}\right)^{2}}\left|\psi_{n}\right\rangle\left\langle\psi_{n^{\prime}}\right|
$$

where the combination of the indices $v$ and $v^{\prime}$ determines the path involved in the process; e.g., when $v=1$ and $v^{\prime}=1$, the hole only hops through $O_{1}$ hence giving $\mathcal{H}_{1}^{O}$. Symmetry leads to the following equivalence relations between $\mathcal{H}_{\ell}^{O}$ corresponding to the processes starting from $\operatorname{Ir}_{2}$ and processes starting from $\operatorname{Ir}_{1}$ ions:

$$
\mathcal{H}_{2}^{O} \sim \mathcal{H}_{7}^{O}, \mathcal{H}_{4}^{O} \sim \mathcal{H}_{3}^{O}, \mathcal{H}_{6}^{O} \sim \mathcal{H}_{5}^{O}, \mathcal{H}_{8}^{O} \sim \mathcal{H}_{1}^{O},
$$

in which the equivalence relation means the equality of matrix elements involving $\psi_{2}$ and $\psi_{3}$ under interchanging $\psi_{2} \mapsto \psi_{3}$ and $\psi_{3} \mapsto \psi_{2}$. For example, $\left\langle\psi_{2}\left|\mathcal{H}_{2}^{O}\right| \psi_{2}\right\rangle=\left\langle\psi_{3}\left|\mathcal{H}_{7}^{O}\right| \psi_{3}\right\rangle,\left\langle\psi_{2}\left|\mathcal{H}_{2}^{O}\right| \psi_{3}\right\rangle=\left\langle\psi_{3}\left|\mathcal{H}_{7}^{O}\right| \psi_{2}\right\rangle$, and so on. Summing up over all paths (both starting at $\operatorname{Ir}_{1}$ and $\mathrm{Ir}_{2}$ ) with the corresponding polarization prefactors in the way given in Eq. (3) leads to the final expression Eq. (26) for the Raman operator $\mathcal{R}_{\langle i j\rangle_{2}}^{\text {med }}$ coming from oxygen-mediated processes.

\section{Raman operator from the processes involving mixed direct and the oxygen-mediated hopping}

The mixed hopping can be viewed as the combination of the direct hopping and the oxygen-mediated hopping, so we can compute $\mathcal{H}_{i}^{m}$ associated with each path as

$$
\mathcal{H}_{\ell}^{m}=\sum_{n, n^{\prime}}\left|\psi_{n}\right\rangle\left\langle\psi_{n}\left|\mathcal{H}_{t} \mathcal{G} \mathcal{H}_{t} \mathcal{G} \mathcal{H}_{t}\right| \psi_{n^{\prime}}\right\rangle\left\langle\psi_{n^{\prime}}\right|=\left\{\begin{array}{l}
\sum_{n, n^{\prime}} \sum_{\mu} \frac{\left(\left\langle D_{\mu}\left|\mathcal{H}_{t}\right| \psi_{n}\right\rangle\right)^{*} \cdot\left(\left\langle D_{\mu}\left|\mathcal{H}_{t}\right| O_{v}\right\rangle\left\langle O_{\nu}\left|\mathcal{H}_{t}\right| \psi_{n^{\prime}}\right\rangle\right)}{\left(2 E_{1 \mathrm{~h}}+\omega_{\mathrm{in}}-\left(E_{2 \mathrm{~h}}+E_{0 \mathrm{~h}}\right)\right)\left(\omega_{\mathrm{in}}-\Delta_{\mathrm{pd}}\right)}\left|\psi_{n}\right\rangle\left\langle\psi_{n^{\prime}}\right| \\
\sum_{n, n^{\prime}} \sum_{\mu} \frac{\left(\left\langle D_{\mu}\left|\mathcal{H}_{t}\right| O_{v}\right\rangle\left\langle O_{\nu}\left|\mathcal{H}_{t}\right| \psi_{n}\right\rangle\right)^{*} \cdot\left(\left\langle D_{\mu}\left|\mathcal{H}_{t}\right| \psi_{n^{\prime}}\right\rangle\right)}{\left(\omega_{\mathrm{in}}-\Delta_{\mathrm{pd}}\right)\left(2 E_{1 \mathrm{~h}}+\omega_{\mathrm{in}}-\left(E_{2 \mathrm{~h}}+E_{0 \mathrm{~h}}\right)\right)}\left|\psi_{n}\right\rangle\left\langle\psi_{n^{\prime}}\right|
\end{array},\right.
$$

where the first case represent the path starting from the oxygen-mediated hopping and the second one from the direct hopping. The overlap matrix elements $\langle\ldots\rangle$ in (A6) are computed either for the direct hopping process or for the oxygen-mediated hopping process, so no additional consideration needs to be taken here. The equivalence relations between the processes starting from $\operatorname{Ir}_{2}$ 
and from $\operatorname{Ir}_{1}$ are given by

$$
\mathcal{H}_{2}^{m} \sim \mathcal{H}_{5}^{m}, \mathcal{H}_{4}^{m} \sim \mathcal{H}_{7}^{m}, \mathcal{H}_{6}^{m} \sim \mathcal{H}_{1}^{m}, \mathcal{H}_{8}^{m} \sim \mathcal{H}_{3}^{m},
$$

where the equivalence has the same meaning as in Eq. (A5) above. Finally, summing up over all paths with the corresponding polarization factors leads to the final expression of Eq. (30) for the Raman operator $\mathcal{R}_{\langle i j\rangle_{z}}^{\operatorname{mix}}$ coming from the mixed processes.

\section{Analytic expressions of the prefactor constants in the Raman operator}

Here we provide the analytic expressions for the prefactor constants entering the Raman operator. For simpler algebra manipulation, the calculations are done using the total- $J$ basis (e.g., $\left|J, J_{z}\right\rangle$ ) instead of the orbital basis. The details of using this basis can be found at [62]. Denoting

$$
\begin{gathered}
f_{1}=-\frac{1}{3}\left(\frac{J_{H}+3\left(U_{1}+3 \lambda-\omega_{\text {in }}\right)}{6 J_{H}^{2}+J_{H}\left(U_{1}+4 \lambda-\omega_{\text {in }}\right)-\left(U_{1}-\omega_{\text {in }}\right)\left(U_{1}+3 \lambda-\omega_{\text {in }}\right)}\right), \\
f_{2}=\frac{4}{3}\left(\frac{3 J_{H}-U_{1}-3 \lambda+\omega_{\text {in }}}{6 J_{H}-2 U_{1}-3 \lambda+2 \omega_{\text {in }}}\right) \frac{J_{H}}{6 J_{H}^{2}-J_{H}\left(8 U_{1}+17 \lambda-8 \omega_{\text {in }}\right)+\left(2 U_{1}+3 \lambda-2 \omega_{\text {in }}\right)\left(U_{1}+3 \lambda-\omega_{\text {in }}\right)}, \\
f_{3}=\frac{7 J_{H}-3 U_{1}-9 \lambda+3 \omega_{\text {in }}}{6 J_{H}^{2}-J_{H}\left(8 U_{1}+17 \lambda-8 \omega_{\text {in }}\right)+\left(2 U_{1}+3 \lambda-2 \omega_{\text {in }}\right)\left(U_{1}+3 \lambda-\omega_{\text {in }}\right)}, \\
f_{4}=\frac{1}{6 J_{H}-2 U_{1}-3 \lambda+2 \omega_{\text {in }}},
\end{gathered}
$$

the various coupling constants take the following form:

$$
\begin{gathered}
J^{(2)}=\frac{4}{9} f_{1}\left(2 t_{1}+t_{3}\right)^{2}-\frac{8}{9} f_{2}\left(9 t_{4}^{2}+2\left(t_{1}-t_{3}\right)^{2}\right), \\
K^{(2)}=\frac{8}{3} f_{2}\left(3 t_{4}^{2}+\left(t_{1}-t_{3}\right)^{2}\right), \\
\Gamma^{(2)}=8 f_{2} t_{4}^{2}, \quad \Gamma^{(2)}=-\frac{8}{3} f_{2} t_{4}\left(t_{1}-t_{3}\right), \\
\Gamma^{(3)}=\frac{16}{3} f_{2} t_{2}\left(t_{1}-t_{3}\right), \quad \Gamma^{\prime(3)}=8 f_{2} t_{2} t_{4}, \\
K^{(4)}=-8 f_{2} t_{2}^{2},
\end{gathered}
$$

where we have introduced $t_{4}$ for the general case with lower bond symmetry (in the main text $t_{4}=0$ ). In the limit $\omega_{\text {in }} \rightarrow 0$, these coupling constants reduce to the superexchange coupling constants for the nearest-neighbor $J-K-\Gamma-\Gamma^{\prime}$ model, and are in agreement with expressions given in Ref. [74]. The remaining coupling constants that are associated with the nonLoudon-Fleury processes take the form:

$$
\begin{aligned}
J^{\prime(4)} & =\frac{16}{9}\left(f_{2}-f_{1}\right) t_{2}^{2}, \\
K^{\prime(4)} & =\frac{8}{9}\left(4 f_{1}-f_{2}\right) t_{2}^{2},
\end{aligned}
$$

$$
\begin{gathered}
h_{\Gamma}^{(3)}=\frac{8}{3}\left(\frac{1}{2} f_{2}+\frac{1}{3} f_{4}\right) t_{2}\left(t_{1}-t_{3}\right), \\
h_{\Gamma}^{\prime(3)}=\frac{1}{3}\left(f_{3}+f_{4}\right) t_{2} t_{4}, \\
\tilde{\Gamma}^{(3)}=-\frac{2}{9}\left(f_{3}-3 f_{4}\right) t_{2} t_{4} .
\end{gathered}
$$

The last two coupling constants, which are associated with $t_{4}$, give rise to the following additional terms in the Raman operator:

$$
\begin{aligned}
& -\frac{1}{2} \sum_{\langle i j\rangle_{v}}\left(P_{d^{\perp} d}-P_{d d^{\perp}}\right) i h_{\Gamma}^{\prime(3)}\left(S_{i}^{\beta_{v}}+S_{i}^{\gamma_{v}}+S_{j}^{\beta_{v}}+S_{j}^{\gamma_{v}}\right) \\
& -\frac{1}{2} \sum_{\langle i j\rangle_{v}}\left(P_{d^{\perp} d}+P_{d d^{\perp}}\right) \tilde{\Gamma}^{(3)}\left(S_{i}^{\alpha_{v}}\left(S_{j}^{\beta_{v}}-S_{j}^{\gamma_{v}}\right)\right. \\
& \left.\quad+\left(S_{i}^{\beta_{v}}-S_{i}^{\gamma_{v}}\right) S_{j}^{\alpha_{v}}\right),
\end{aligned}
$$

which are ignored in the main text where $t_{4}=0$.
[1] T. P. Devereaux and R. Hackl, Rev. Mod. Phys. 79, 175 (2007).

[2] B. S. Shastry and B. I. Shraiman, Phys. Rev. Lett. 65, 1068 (1990).

[3] B. S. Shastry and B. I. Shraiman, Int. J. Mod. Phys. B 5, 365 (1991).

[4] A. V. Chubukov and D. M. Frenkel, Phys. Rev. Lett. 74, 3057 (1995).

[5] A. V. Chubukov and D. M. Frenkel, Phys. Rev. B 52, 9760 (1995).

[6] L. Benfatto, M. B. Silva Neto, A. Gozar, B. S. Dennis, G. Blumberg, L. L. Miller, S. Komiya, and Y. Ando, Phys. Rev. B 74, 024416 (2006).
[7] O. Cépas, J. O. Haerter, and C. Lhuillier, Phys. Rev. B 77, 172406 (2008).

[8] W.-H. Ko, Z.-X. Liu, T.-K. Ng, and P. A. Lee, Phys. Rev. B 81, 024414 (2010).

[9] D. Wulferding, P. Lemmens, P. Scheib, J. Röder, P. Mendels, S. Chu, T. Han, and Y. S. Lee, Phys. Rev. B 82, 144412 (2010).

[10] N. Perkins and W. Brenig, Phys. Rev. B 77, 174412 (2008).

[11] N. B. Perkins, G.-W. Chern, and W. Brenig, Phys. Rev. B 87, 174423 (2013).

[12] K. Sen, Y. Yao, R. Heid, A. Omoumi, F. Hardy, K. Willa, M. Merz, A. A. Haghighirad, and M. Le Tacon, Phys. Rev. B 100, 104301 (2019). 
[13] L. J. Sandilands, Y. Tian, K. W. Plumb, Y.-J. Kim, and K. S. Burch, Phys. Rev. Lett. 114, 147201 (2015).

[14] L. J. Sandilands, Y. Tian, A. A. Reijnders, H.-S. Kim, K. W. Plumb, Y.-J. Kim, H.-Y. Kee, and K. S. Burch, Phys. Rev. B 93, 075144 (2016).

[15] H. Gretarsson, N. H. Sung, M. Höppner, B. J. Kim, B. Keimer, and M. Le Tacon, Phys. Rev. Lett. 116, 136401 (2016).

[16] A. Glamazda, P. Lemmens, S.-H. Do, Y. S. Choi, and K.-Y. Choi, Nat. Commun. 7, 12286 (2016).

[17] A. Sahasrabudhe, D. A. S. Kaib, S. Reschke, R. German, T. C. Koethe, J. Buhot, D. Kamenskyi, C. Hickey, P. Becker, V. Tsurkan, A. Loidl, S. H. Do, K. Y. Choi, M. Grüninger, S. M. Winter, Z. Wang, R. Valentí, and P. H. M. van Loosdrecht, Phys. Rev. B 101, 140410(R) (2020).

[18] D. Wulferding, Y. Choi, S.-H. Do, C. H. Lee, P. Lemmens, C. Faugeras, Y. Gallais, and K.-Y. Cho, Nat. Commun. 11, 1603 (2020).

[19] Y. Wang, G. B. Osterhoudt, Y. Tian, P. Lampen-Kelley, A. Banerjee, T. Goldstein, J. Yan, J. Knolle, H. Ji, R. J. Cava, J. Nasu, Y. Motome, S. E. Nagler, D. Mandrus, and K. S. Burch, npj Quantum Mater. 5, 14 (2020).

[20] J. Knolle, G.-W. Chern, D. L. Kovrizhin, R. Moessner, and N. B. Perkins, Phys. Rev. Lett. 113, 187201 (2014).

[21] B. Perreault, J. Knolle, N. B. Perkins, and F. J. Burnell, Phys. Rev. B 92, 094439 (2015).

[22] B. Perreault, J. Knolle, N. B. Perkins, and F. J. Burnell, Phys. Rev. B 94, 060408(R) (2016).

[23] B. Perreault, J. Knolle, N. B. Perkins, and F. J. Burnell, Phys. Rev. B 94, 104427 (2016).

[24] J. Fu, J. G. Rau, M. J. P. Gingras, and N. B. Perkins, Phys. Rev. B 96, 035136 (2017).

[25] I. Rousochatzakis, S. Kourtis, J. Knolle, R. Moessner, and N. B. Perkins, Phys. Rev. B 100, 045117 (2019).

[26] G. Cao and L. DeLong (eds.), Frontiers of 4d-and 5d-Transition Metal Oxides (World Scientific Publishing, Singapore, 2013).

[27] W. Witczak-Krempa, G. Chen, Y. B. Kim, and L. Balents, Annu. Rev. Condens. Matter Phys. 5, 57 (2014).

[28] J. G. Rau, E. K.-H. Lee, and H.-Y. Kee, Annu. Rev. Condens. Matter Phys. 7, 195 (2016).

[29] J. Nasu, J. Knolle, D. Kovrizhin, Y. Motome, and R. Moessner, Nat. Phys. 12, 912 (2016).

[30] C. Ulrich, G. Khaliullin, M. Guennou, H. Roth, T. Lorenz, and B. Keimer, Phys. Rev. Lett. 115, 156403 (2015).

[31] Y. Gim, A. Sethi, Q. Zhao, J. F. Mitchell, G. Cao, and S. L. Cooper, Phys. Rev. B 93, 024405 (2016).

[32] H. Gretarsson, J. Sauceda, N. H. Sung, M. Höppner, M. Minola, B. J. Kim, B. Keimer, and M. Le Tacon, Phys. Rev. B 96, 115138 (2017).

[33] S.-M. Souliou, J. Chaloupka, G. Khaliullin, G. Ryu, A. Jain, B. J. Kim, M. Le Tacon, and B. Keimer, Phys. Rev. Lett. 119, 067201 (2017).

[34] A. Metavitsiadis, W. Natori, J. Knolle, and W. Brenig (unpublished).

[35] P. A. Fleury and R. Loudon, Phys. Rev. 166, 514 (1968).

[36] R. J. Elliott and R. Loudon, Phys. Lett. 3, 189 (1963).

[37] Yiping Wang and Kenneth Burch (private communication).

[38] S. Trebst, arXiv:1701.07056.
[39] H. Takagi, T. Takayama, G. Jackeli, G. Khaliullin, and S. E. Nagler, Nat. Rev. Phys. 1, 264 (2019).

[40] Y. Motome and J. Nasu, J. Phys. Soc. Jpn. 89, 012002 (2020).

[41] Y. Singh and P. Gegenwart, Phys. Rev. B 82, 064412 (2010).

[42] S. Hwan Chun, J.-W. Kim, J. Kim, H. Zheng, C. C. Stoumpos, C. D. Malliakas, J. F. Mitchell, K. Mehlawat, Y. Singh, Y. Choi, T. Gog, A. Al-Zein, M. M. Sala, M. Krisch, J. Chaloupka, G. Jackeli, G. Khaliullin, and B. J. Kim, Nat. Phys. 11, 462 (2015).

[43] S. C. Williams, R. D. Johnson, F. Freund, S. Choi, A. Jesche, I. Kimchi, S. Manni, A. Bombardi, P. Manuel, P. Gegenwart, and R. Coldea, Phys. Rev. B 93, 195158 (2016).

[44] K. W. Plumb, J. P. Clancy, L. J. Sandilands, V. V. Shankar, Y. F. Hu, K. S. Burch, H.-Y. Kee, and Y.-J. Kim, Phys. Rev. B 90, 041112(R) (2014).

[45] J. A. Sears, M. Songvilay, K. W. Plumb, J. P. Clancy, Y. Qiu, Y. Zhao, D. Parshall, and Y.-J. Kim, Phys. Rev. B 91, 144420 (2015).

[46] A. Banerjee, J. Yan, J. Knolle, C. A. Bridges, M. B. Stone, M. D. Lumsden, D. G. Mandrus, D. A. Tennant, R. Moessner, and S. E. Nagler, Science 356, 1055 (2017).

[47] Y. Kasahara, T. Ohnishi, Y. Mizukami, O. Tanaka, S. Ma, K. Sugii, N. Kurita, H. Tanaka, J. Nasu, Y. Motome, T. Shibauchi, and Y. Matsuda, Nature (London) 559, 227 (2018).

[48] A. Biffin, R. D. Johnson, S. Choi, F. Freund, S. Manni, A. Bombardi, P. Manuel, P. Gegenwart, and R. Coldea, Phys. Rev. B 90, 205116 (2014).

[49] A. Ruiz, A. Frano, N. P. Breznay, I. Kimchi, T. Helm, I. Oswald, J. Y. Chan, R. Birgeneau, Z. Islam, and J. G. Analytis, Nat. Commun. 8, 961 (2017).

[50] M. Majumder, F. Freund, T. Dey, M. Prinz-Zwick, N. Büttgen, Y. Skourski, A. Jesche, A. A. Tsirlin, and P. Gegenwart, Phys. Rev. Mater. 3, 074408 (2019).

[51] A. Biffin, R. D. Johnson, I. Kimchi, R. Morris, A. Bombardi, J. G. Analytis, A. Vishwanath, and R. Coldea, Phys. Rev. Lett. 113, 197201 (2014).

[52] K. Modic, T. Smidt, I. Kimchi, N. Breznay, A. Biffin, S. Choi, R. Johnson, R. Coldea, P. Watkins-Curry, G. McCandless, J. Chan, F. Gandara, A. Vishwanath, A. Shekhter, R. Mcdonald, and J. Analytis, Nat. Commun. 5, 4203 (2014).

[53] V. Y. Yushankhai and R. Hayn, Europhys. Lett. 47, 116 (1999).

[54] V. M. Katukuri, S. Nishimoto, V. Yushankhai, A. Stoyanova, H. Kandpal, S. Choi, R. Coldea, I. Rousochatzakis, L. Hozoi, and J. van den Brink, New J. Phys. 16, 013056 (2014).

[55] J. G. Rau, Eric Kin-Ho Lee, and H.-Y. Kee, Phys. Rev. Lett. 112, 077204 (2014).

[56] Eric Kin-Ho Lee and Y. B. Kim, Phys. Rev. B 91, 064407 (2015).

[57] Eric Kin-Ho Lee, J. G. Rau, and Y. B. Kim, Phys. Rev. B 93, 184420 (2016).

[58] I. Rousochatzakis and N. B. Perkins, Phys. Rev. Lett. 118, 147204 (2017).

[59] S. M. Winter, A. A. Tsirlin, M. Daghofer, J. van den Brink, Y. Singh, P. Gegenwart, and R. Valentí, J. Phys.: Condens. Matter 29, 493002 (2017).

[60] G. Jackeli and G. Khaliullin, Phys. Rev. Lett. 102, 017205 (2009).

[61] J. Chaloupka, G. Jackeli, and G. Khaliullin, Phys. Rev. Lett. 105, 027204 (2010). 
[62] N. B. Perkins, Y. Sizyuk, and P. Wölfle, Phys. Rev. B 89, 035143 (2014).

[63] Y. Sizyuk, C. Price, P. Wölfle, and N. B. Perkins, Phys. Rev. B 90, 155126 (2014).

[64] D. J. Klein, J. Chem. Phys. 61, 786 (1974).

[65] S. Ducatman, I. Rousochatzakis, and N. B. Perkins, Phys. Rev. B 97, 125125 (2018).

[66] I. Rousochatzakis and N. B. Perkins, Phys. Rev. B 97, 174423 (2018).

[67] M. Li, I. Rousochatzakis, and N. B. Perkins, Phys. Rev. Research 2, 013065 (2020).

[68] M. Li, I. Rousochatzakis, and N. B. Perkins, Phys. Rev. Research 2, 033328 (2020).
[69] A. Ruiz, V. Nagarajan, M. Vranas, G. Lopez, G. T. McCandless, I. Kimchi, J. Y. Chan, N. P. Breznay, A. Frañó, B. A. Frandsen, and J. G. Analytis, Phys. Rev. B 101, 075112 (2020).

[70] H.-S. Kim, E. K.-H. Lee, and Y. B. Kim, Europhys. Lett. 112, 67004 (2015).

[71] B. Perreault, Identifying a Kitaev spin liquid, Ph.D. thesis, University of Minnesota, 2016.

[72] W. M. H. Natori, R. Moessner, and J. Knolle, Phys. Rev. B 100, 144403 (2019).

[73] N. Arakawa and K. Yonemitsu, Phys. Rev. B 103, L100408 (2021).

[74] S. M. Winter, Y. Li, H. O. Jeschke, and R. Valentí, Phys. Rev. B 93, 214431 (2016). 